\title{
Effect of external pulsation on kinematics of fluid particles in the field of Lamb-Oseen vortex pair
}

\author{
S ANANTH PAI*, SHALIGRAM TIWARI and T SUNDARARAJAN \\ Department of Mechanical Engineering, Indian Institute of Technology Madras, Chennai 600 036, India \\ e-mail: sudaananth@gmail.com
}

MS received 30 April 2016; revised 1 September 2016; accepted 3 October 2016

\begin{abstract}
The effect of external pulsation on a pair of stationary Lamb-Oseen vortices of equal strength has been analyzed to investigate kinematic behavior of a fluid particle. The assumption of vortices being treated stationary or fixed vortex filaments is valid in a reference frame attached to the vortex system with axes along and perpendicular to the line of their centers. Also, it is assumed that change in core shape and size is much small, with least possibility of core merger. In such situations, periodic particle paths are observed and superposition of pulsation becomes beneficial. In the present work, motion of a representative fluid particle is modeled as a non-linear dynamical system by varying both amplitude and frequency of external pulsation. Effect of external pulsation has been brought out with the help of quantification of deviation from periodic paths by using the concept of total average deviation. Results are presented in terms of particle paths, velocity phase plots, velocity signals and their spectra for varying amplitude and frequency of external pulsation.
\end{abstract}

Keywords. Lamb-Oseen vortex pair; stationary vortices; nonlinear dynamical system; particle paths; external pulsation.

\section{Introduction}

Transport and mixing in fluid flow is influenced by the presence of vortices. In two-dimensional flows vortices can be modeled as inviscid, Rankine or Lamb-Oseen vortices [1]. These vortex models are exact solutions of the NavierStokes equations under different assumptions. In general, these vortices move under the influence of each other. Initial contributions to motion of vortices by Helmholtz, Kirchoff and Gröbli have been compiled by Aref [2]. The use of inviscid vortices (also referred to as 'point vortex' in two-dimensions) to analyze flow phenomena like von Karman vortex street, vortex rings, rolling up of vortex sheets, etc. has been well documented in classical books of fluid mechanics and vortex dynamics [3, 4]. In the case of point vortices, the velocity decreases as inverse function of radius. A real vortex in a flow possesses vorticity around its center. In order to simplify the analysis, one may assume the core region of vorticity to have circular shape and the vorticity to be uniformly distributed in the core. Such a vortex with uniform vorticity in a circular core is called a Rankine vortex [1]. In the case of Rankine vortex the velocity increases linearly inside the core while it decreases as inverse function of radius outside the core. Rankine vortex models have been used to analyze heat transfer and mixing due to fluid flows [5-7]. Vortices in actual flow situations exhibit diffusion of vorticity in the core due to the effect of viscosity. Hence, there takes place an increase in the core size with time. Such a vortex accounting for effect of diffusion is modeled as Lamb-Oseen vortex [1]. The Lamb-Oseen vortex model considers vorticity diffusion from an initially inviscid vortex configuration where the vorticity tends to be infinitely large near the center. This leads to a Gaussian vorticity distribution in the core at a given time $(t>0)$ and is expressed as

$$
\omega=\frac{\Gamma}{4 \pi v t} e^{-r^{2} / 4 v t}
$$

where $\omega$ is the vorticity at any radial location $(r), \Gamma$ is the circulation and $v$ is the kinematic viscosity. The tangential velocity, $V_{\theta}$, is given by

$$
V_{\theta}=\frac{\Gamma}{2 \pi r}\left(1-e^{-r^{2} / 4 v t}\right)
$$

The radial distance at which the velocity becomes maximum can be assumed to be the radius of the core. It can be shown that the maximum velocity occurs at a radial distance of nearly $\sqrt{5 v t}$ [8]. Lamb-Oseen vortex models closely mimic coherent vortex structures in two-dimensional turbulent flows [9]. One of the major phenomenon involved with Lamb-Oseen vortices is the merger of vortex cores [10-12].

*For correspondence 
The motion of vortices due to influence of each other can be modeled as a dynamical system $[13,14]$. To analyze the fluid particle motion, any particles can be assumed as a vortex having zero circulation [15]. Such a dynamical system can be used to analyze wake vortices behind a bluff body [14], to study transport of particles in turbulent flows [16] and many other applications. Stationary solution of a dynamical system is referred to as 'equilibrium point' (EP) which is analogous to stagnation zones in fluid mechanics. EPs can be classified based on the characteristics of phase paths around them as hyperbolic or elliptic. In a fluid flow situation, presence of elliptic points is not desired for efficient mixing. The presence of hyperbolic points such as a saddle assists in stretching and folding of material lines which in turn is helpful for efficient mixing [17]. In addition to the presence of hyperbolic points, superposition of external pulsations can improve mixing and transport characteristics a flow domain. External pulsations are well utilized to increase mixing characteristics in micro-fluidic devices by using acoustic, electrical or magnetic means [18]. Effect of external pulsations on the wake behind circular cylinders on vortex shedding behavior has been studied by Lu and Papadakis [19].

The pair of Lamb-Oseen vortices in a fluid move under the influence of each other. In the case of equal strength vortices, co-rotating vortices rotate about an axis that is perpendicular to the line of centers and counter rotating vortices translate. The angular velocity about the rotation axis of the co-rotating system and the translational velocity of the counter-rotating system do not vary with time [20]. During rotation or translation, the relative distance between the vortices does not change when there is least possibility of core merger. If a rotating frame of reference is attached to the co-rotating pair or a translating frame of reference is attached to the counter-rotating pair, the vortex system can be considered stationary in the respective frame of reference.

In the present work, a pair of stationary Lamb-Oseen vortices are considered and the particle paths generated by these vortices are analyzed. Even though there exist many parametric combinations of strengths and distance between the vortices, the present work assumes equal strength for the considered vortex pair. For the system of moving vortices, the initial distance between the vortices can be assumed to be four times the core radius to avoid the possibility of core merger [21]. In Lamb-Oseen system, the core radius is normally variable and hence for given initial separation the growing core radius will not satisfy the criteria for non-merger. Moreover, there is a better possibility of mixing during core merger due to which superposition of external pulsation may be less effective. Consequently, the effect of external pulsation becomes mostly important in non-merging vortex systems. Therefore, present work considers the situation when change in core radius is much small (may be true at large core radius after long time) and it can be assumed to be almost independent of time. Such an attempt for analysis is expected to cause less error while still successfully being able to bring out the effect of external pulsation. Both the cases of co-rotating and counter-rotating vortex pair have been separately analyzed. On either of these arrangements, external pulsation has been superimposed. An attempt is made to examine the effect of external pulsation on particle paths, $u$-velocity signal and its Fourier spectra. The governing equations form a dynamical system which have been solved using RK-4 method.

\section{Problem statement}

In a vortex dominated flow, the interactions of vortices influence the transport characteristics. An interaction between two vortices may be assumed as building block or elementary form of interaction. Even though vortices move relative to each other, analysis by considering the vortices as stationary can be useful to understand transport of passive particles. Every fluid particle under the influence of stationary vortices moves in its own unique way. Stationary vortices produce a large number of periodic paths. Periodic particle motion is not quite favorable when mixing is desired in the flow domain. In fact, mixing can be enhanced if the particles possess higher degree of wandering in the flow domain. In order to increase wandering of fluid particles, one may impose fluctuations to the velocity field in the form of external pulsation. In the present work, influence of external sinusoidal pulsations has been studied.

A system of two stationary Lamb-Oseen vortices of equal strength in an unbounded fluid domain is shown in figure 1 . Both co-rotating and counter-rotating arrangements of vortices have been considered for analysis by assuming them stationary or as fixed vortex filaments. Such an assumption is valid in a rotating frame attached to co-rotating pair or translating frame attached to counter-rotating pair. The effect of external pulsation on particle paths has been studied by superposition of a sinusoidal oscillation on the field generated by the vortices. Even though the pulsation can be superimposed in both $x$ - and $y$-directions in general, the present work considers only the effect of pulsation along $x$-direction.

\section{Governing equations}

The vorticity at the center of a point vortex (inviscid) tends to become infinitely large. When diffusion of vorticity with time is considered, the resulting vortex forms the LambOseen vortex. Figure $2 \mathrm{a}$ and $\mathrm{b}$ present the variation of vorticity and velocity respectively as function of radial distance. It is apparent that maximum values of both velocity and vorticity decrease with increase in time. This is due to vorticity diffusion and increase in core radius. For a Lamb-Oseen vortex, the velocity becomes maximum at the end of the core radius as observed for a Rankine vortex. 


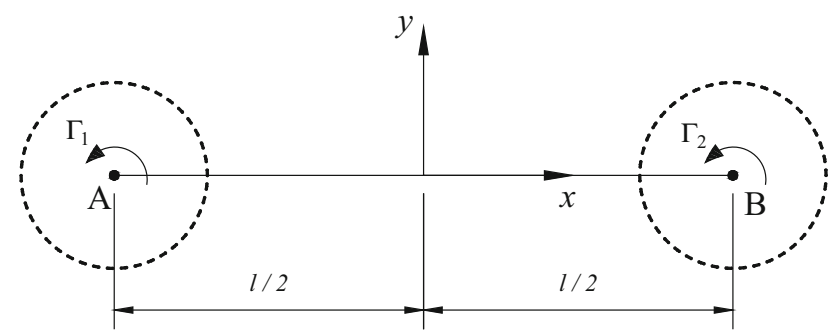

Figure 1. Lamb-Oseen vortex pair with the dotted line representing core radius.

Equation (2) can be written in the Cartesian coordinate system as

$$
\begin{aligned}
& u=\frac{-\Gamma y}{2 \pi\left(x^{2}+y^{2}\right)}\left(1-e^{-\frac{x^{2}+y^{2}}{4 v t}}\right) \\
& v=\frac{\Gamma x}{2 \pi\left(x^{2}+y^{2}\right)}\left(1-e^{-\frac{x^{2}+y^{2}}{4 v t}}\right)
\end{aligned}
$$

where $u$ is the $x$-component of the velocity and $v$ is the $y$ component of the velocity.

When two vortices are simultaneously present in the domain, the velocity induced because of individual vortices can be superimposed. Referring to figure 1, the Cartesian components of velocity of a particle in the flow domain are given by,

$$
\frac{d x}{d t}=u=-\frac{\Gamma_{1} y}{2 \pi} \frac{\left(1-e^{-\frac{(x+l / 2)^{2}+y^{2}}{4 v t}}\right)}{(x+l / 2)^{2}+y^{2}}-\frac{\Gamma_{2} y}{2 \pi} \frac{\left(1-e^{-\frac{(x-l / 2)^{2}+y^{2}}{4 v t}}\right)}{(x-l / 2)^{2}+y^{2}}
$$

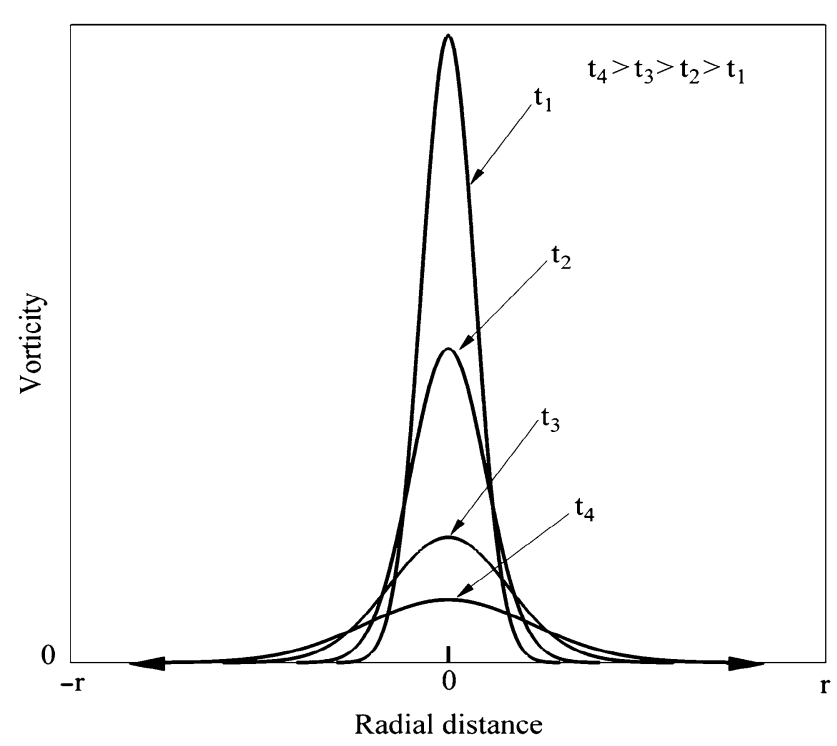

(a)

$$
\begin{aligned}
\frac{d y}{d t}= & v \\
= & \frac{\Gamma_{1}}{2 \pi} \frac{(x+l / 2)\left(1-e^{-\frac{(x+l / 2)^{2}+y^{2}}{40 t}}\right)}{(x+l / 2)^{2}+y^{2}} \\
& +\frac{\Gamma_{2}}{2 \pi} \frac{(x-l / 2)\left(1-e^{-\frac{(x-l / 2)^{2}+y^{2}}{40 t}}\right)}{(x-l / 2)^{2}+y^{2}}
\end{aligned}
$$

The above expressions can be non-dimensionalized with respect to the core radius $r_{c}$ for length variables and $\tau=$ $2 \pi r_{c}^{2} / \Gamma$ for time. Let $r *=r / r_{c}, x *=x / r_{c}, y *=y / r_{c}$, $l *=l / r_{c}, \quad \Gamma_{1}=\alpha \Gamma, \Gamma_{2}=\beta \Gamma$, and $t *=t / \tau$. To avoid merging, the distance between vortices has to be at least four times the core radius [9] and hence the distance between the vortex centers is taken as $l=4 r_{c}$. The resulting non-dimensionalized equations after removing superscript '*' are given by

$$
\begin{aligned}
\frac{d x}{d t} & =u \\
& =-y\left(\frac{\alpha\left(1-e^{-\alpha r_{1}^{2} \operatorname{Re} /(8 \pi \mathrm{t})}\right)}{r_{1}^{2}}+\frac{\beta\left(1-e^{-\beta r_{2}^{2} \operatorname{Re} /(8 \pi \mathrm{t})}\right)}{r_{2}^{2}}\right)
\end{aligned}
$$

$$
\begin{aligned}
& \frac{d y}{d t}=v \\
& =\left(\frac{\alpha(x+2)\left(1-e^{-\alpha r_{1}^{2} \operatorname{Re} /(8 \pi \mathrm{t})}\right)}{r_{1}^{2}}+\frac{\beta(x-2)\left(1-e^{-\beta r_{2}^{2} \operatorname{Re} /(8 \pi \mathrm{t})}\right)}{r_{2}^{2}}\right)
\end{aligned}
$$

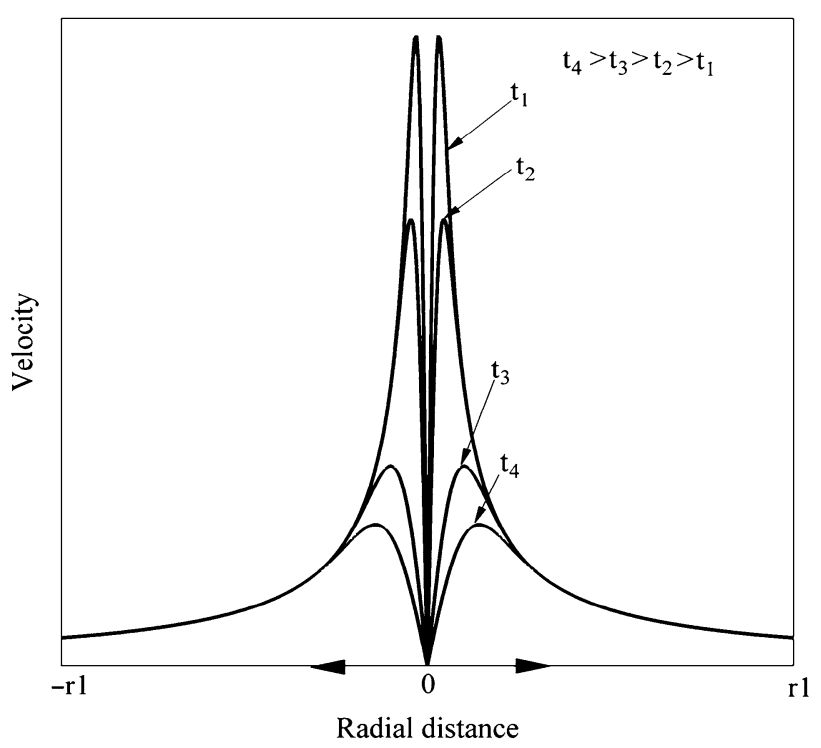

(b)

Figure 2. Variation of (a) vorticity and (b) viscosity with time. 

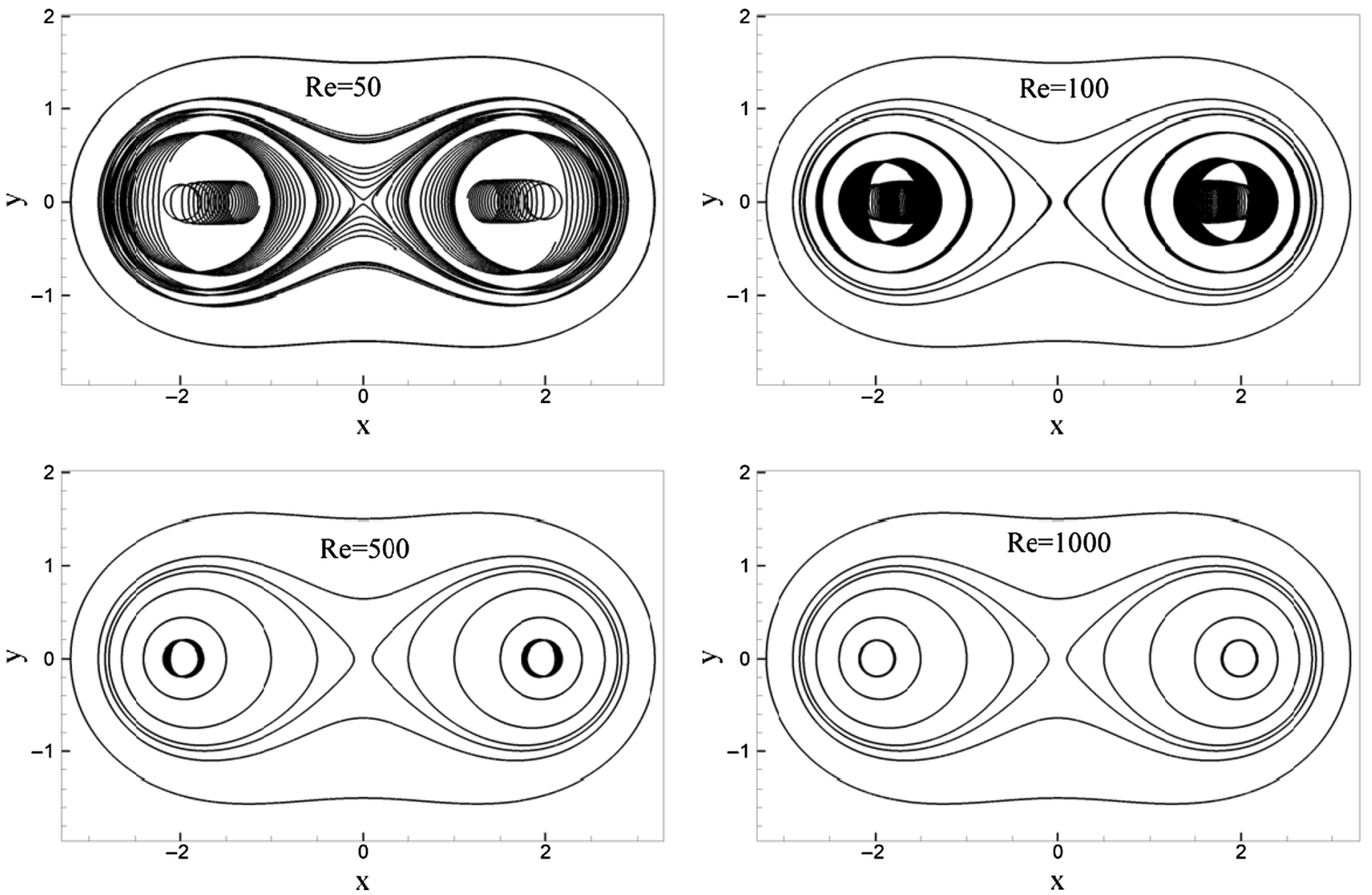

Figure 3. Particles paths for different values of Re.

where $\operatorname{Re}=\Gamma / v$ is the Reynolds number, $r_{1}=$ $\sqrt{(x+2)^{2}+y^{2}}$ and $r_{2}=\sqrt{(x-2)^{2}+y^{2}}$. Equations (6a) and (6b) are solved simultaneously using RK-4 method for different initial positions of the particles in case of co-rotating vortices $(\alpha=1$ and $\beta=1)$. For $\operatorname{Re}=50$ the particles are not closed curves and the co-ordinates of the considered initial locations (x, y) are, $(0, \pm 0.1),(0, \pm 1.8),(0, \pm 2.9)$, and (0, $\pm 3.2)$. For $R e=100$ the initial positions are $(0, \pm 0.1),(0$, $\pm 1),(0, \pm 0.5),(0, \pm 1.8),(0, \pm 2.9)$, and $(0, \pm 3.2)$. For large values of $\operatorname{Re}$ (that is $\operatorname{Re}=500$ and $\operatorname{Re}=1000$ ) particle paths are closed curves and they cross $x$-axis at two locations. The initial point assumed at any of these locations gives the same particle path. The time step is chosen to be $\Delta t=0.005$, because for smaller time steps particle paths remain the same. The results obtained are as shown in figure 3 .

It is seen that for $\mathrm{Re}=50$, the particle paths are not closed curves. This may be because of the core radius changing rapidly due to the effect of viscosity that is large at low Reynolds number. For higher values of Re, the particle paths are closed curves. In case the core radius changes with time rapidly, the chances of the ratio of distance between vortex centers and the core radius becoming less than four are high and this may cause deformations in assumed shape of vortex cores. At the onset of merger, assumption of circular core gets flawed and also the assumption of fixed filaments is not quite appropriate. Further, if the particle paths are not closed curves, flow already has built-in possibility of better mixing due to which superimposing pulsation may not be required. Therefore, advantage of analyzing the effect of pulsation is more for the cases where there is almost no core deformation. Accordingly, in the present work, the core radius has been assumed to be a constant. For constant core radius $\left(r_{c}\right)$, nondimensionalization of Eqs. (4) and (5) with respect to the characteristic linear dimension $\left(r_{c}\right)$ while keeping all the other non-dimensionalizing factors same as in Eq. (6), gives

$$
\frac{d x}{d t}=u=-y\left(\frac{\alpha\left(1-e^{-1.25 r_{1}^{2}}\right)}{r_{1}^{2}}+\frac{\beta\left(1-e^{-1.25 r_{2}^{2}}\right)}{r_{2}^{2}}\right)
$$

$$
\begin{aligned}
\frac{d y}{d t} & =v \\
& =\left(\frac{\alpha(x+2)\left(1-e^{-1.25 r_{1}^{2}}\right)}{r_{1}^{2}}+\frac{\beta(x-2)\left(1-e^{-1.25 r_{2}^{2}}\right)}{r_{2}^{2}}\right) .
\end{aligned}
$$


For deriving the above expressions, in the exponential functions of Eqs. (4) and (5), the core radius has been substituted as $r_{c}=\sqrt{5 v t}$ [8]. When pulsation is superimposed in the $x$-direction, Eq. (7) becomes

$$
\begin{aligned}
\frac{d x}{d t}= & -y\left(\frac{\alpha\left(1-e^{-1.25 r_{1}^{2}}\right)}{r_{1}^{2}}+\frac{\beta\left(1-e^{-1.25 r_{2}^{2}}\right)}{r_{2}^{2}}\right) \\
& +A \cos (2 \pi f t)
\end{aligned}
$$

where $A$ and $f$ are the non-dimensionalized amplitude and frequency, respectively. Amplitude of the velocity signal is non-dimensionalized with respect to the maximum tangential velocity and the frequency of pulsation is non-dimensionalized by the circulation frequency of a fluid particle placed on the core boundary of either vortex.

\section{Methodology}

\subsection{Particle motion}

Motion of a particle in two dimensions is associated with four variables, viz., two position variables and two momentum variables. The momentum variables are constant multiples of the velocity variables. Here $u$ and $v$ represent the velocity component along $x$ and $y$-directions respectively. The variation of position variables with time is represented by the particle path in the $x-y$ plane. Variation of momentum variables with time is shown by a Lissajous figure showing the two associated velocities along the $x$ and $y$ axes, respectively. To compute the particle paths, Eqs. (7a) and (7b) are solved simultaneously using RK-4 method in absence of external pulsation and Eqs. (7b) and (8) in the presence of pulsation. The total time for which the integration is carried out is $T=100$ and time step is taken as $\Delta t=0.005$. In every case it was seen that smaller time step did not yield any difference in the particle paths. The initial positions are chosen in such a way that they represent prominent types of particle paths. The pulsation is added to the vortex field as shown in the Eq. (8). The amplitudes of the pulsation chosen for analysis lie between $A=0.1$ and $A=0.5$ and frequencies are varied keeping the amplitude constant. The Fourier spectra of the velocity component in the $x$-direction are utilized to analyze the effect of pulsation.

\subsection{Analysis of equilibrium points}

Equilibrium points (EPs) are the locations in the domain where there is no flow. These points can be evaluated by equating Eqs. (7a) and (7b) to zero and then solving them simultaneously to obtain the coordinates of EP, i.e. $x_{e q}$ and $y_{e q}$. Linearizing the dynamical system near an EP can be helpful in evaluating its nature. The eigen values of the
Jacobian of the dynamical system at EP can predict the nature of the EP in most of the cases where linear approximation is considered to be a good approximation. The Jacobian of the dynamical system is given by

$$
J=\left.\left(\begin{array}{ll}
\frac{\partial u(x, y)}{\partial x} & \frac{\partial u(x, y)}{\partial y} \\
\frac{\partial v(x, y)}{\partial x} & \frac{\partial v(x, y)}{\partial y}
\end{array}\right)\right|_{\left(x_{e q}, y_{e q}\right)} .
$$

Based on the eigen values of the $J$, the nature of EPs can be predicted. There are different types of EPs such as a center, saddle, source, sink or a spiral [22].

\subsection{Quantification of deviation - total average deviation (TAD)}

The deviation of the particle paths from periodicity when the pulsation is superimposed is quantified by using total average deviation (TAD) computed as below. At a particular time $t$, if the position of the particle in the case where no pulsation is superimposed is given by $(x(t), y(t))$ and when the pulsation is applied be given by $(x p(t), y p(t))$. Then the deviation from the periodic path is given by

$$
D=\sqrt{(x(t)-x p(t))^{2}+(y(t)-y p(t))^{2}}
$$

and the total average deviation is given by

$$
T A D=\frac{D}{n}
$$

where $n$ is the number of samples taken.

\section{Results and discussion}

\subsection{Co-rotating vortices}

5.1a Co-rotating vortices without pulsation: In non-pulsating case of co-rotating vortices, three stagnation points are observed which have been presented in table 1 . There exist one saddle and two centers. Some of the representative particle paths generated are shown in figure 4 .

It can be seen that all the particle paths are periodic. There are two major types of particle paths seen in figure 4, the paths of particles that revolve around a single vortex and the ones that revolve around both the vortices. This topology of particle paths for co-rotating vortices matches with those of earlier authors $[10,21]$. If the initial particle position is taken along the $x$-axis, it can be seen that all the prominent types of particle paths are represented. Also since the particle paths are symmetric about the $y$-axis, taking the initial positions only on one side of the $x$-axis will suffice. Hence, for the purpose of analysis, the initial positions are taken along $x$-axis. As discussed earlier, any 
Table 1. EPs location and their nature for co-rotating vortices.

\begin{tabular}{lcc}
\hline Co-rotating case: $\alpha=1$ and $\beta=1$ & \\
\hline $\operatorname{EPs}\left(x_{e q}, y_{e q}\right)$ & Eigen values & Nature of the EP \\
\hline$(0,0)$ & \pm 0.47 & Saddle \\
$(-1.782,0)$ & $\pm 0.71 i$ & Center \\
$(1.782,0)$ & $\pm 0.71 i$ & Center \\
\hline
\end{tabular}

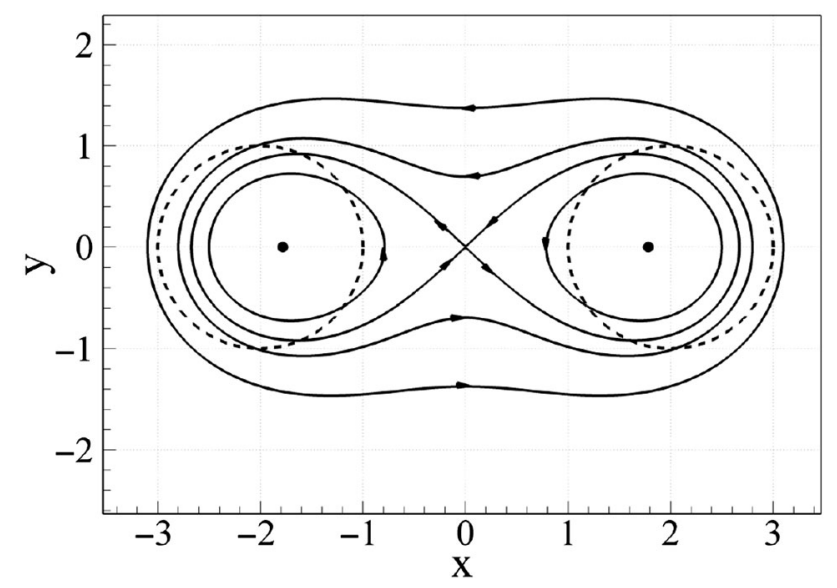

Figure 4. Phase portrait showing the particle paths for corotating Lamb-Oseen vortices.

particle motion is associated with position and momentum variables. The position variables are depicted on the physical $x-y$ plane and momentum is depicted on the $u-v$ plane (Lissajous curve). Fourier spectra give us insights into the periodicity and the frequency of periodic motion. Since in the present work, the fluctuations are imposed on the $u$-velocity field, the Fourier spectral analysis is carried out on the $u$-velocity signal.

The particle paths, the Lissajous curves, the $u$-velocity signal and the Fourier spectra of the $u$-velocity signal have been shown in three dimensions with the third dimension represented by the initial position of particles on the positive $x$-axis in figure 5. The lowest dominant frequency corresponding to the $u$-velocity signals for the particle motion starting from various initial positions is represented in table 2. The other dominant frequencies are the rational multiples of the lowest dominant frequency and hence the motion is periodic. If the paths deviate from periodicity then the ratio of frequencies will not be rational [23].

$5.1 \mathrm{~b}$ Co-rotating vortices with pulsation: The particle paths generated by co-rotating Lamb-Oseen vortices are periodic and this type of particle motion is not desired when good mixing is expected in the flow domain. To make the fluid particles wander more in the flow domain, an external oscillation can be superimposed. In this regard, investigation is carried out on the co-rotating Lamb-Oseen vortices when a pulsation of amplitude $A$ and frequency $f$ is superimposed on the velocity field in the $x$-direction. Although the parametric combinations of amplitude and frequency of pulsations can be large, in the present work, amplitudes between 0.1 and 0.5 are taken, and the frequencies between 0.1 and 3.0 are taken and analysis is carried out. The deviation from the periodic paths shown in figure 5 can be quantified using the parameter $T A D$. The variation of $T A D$ with superimposed pulsation of amplitude 0.1 and different frequencies is depicted in figure 6 .

It can be observed that for small frequencies, the value of $T A D$ is the highest for initial positions far away from the origin on the $x$-axis. These are points that are far away on the right of the vortex system. Particles in these positions traverse periodic paths with low frequencies. Hence when low frequency pulsations are superimposed the deviation from periodic paths is large. It is also seen from figure 6 that the frequency of value 1.0 shows the maximum deviations from periodic paths for the particles located between points $(0,0)$ and $(0,3)$. This is because the periodic paths in this range rotate with frequency $(f)$ close to 1.0 when pulsations are not superimposed. Pulsations of frequency 1.0 cause resonance and therefore a large deviations from the periodic paths can be observed. For frequency values greater than 1 , it is observed that the deviations are small. For particles located near the saddle point $(0,0)$, large values of $T A D$ is observed. This is because a small pulsation of any frequency is adequate to make a particle that is revolving around one vortex to move around both vortices.

Figure 7 shows the particle paths, Lissajous curves, signals of $u$-velocity and their Fourier spectra when pulsations of frequency $f=0.1$ and amplitude $A=0.1$ is superimposed. It may be observed that the particle located at $(0.1,0)$ that was revolving around one vortex (figure 5) is now revolving around both vortices. Also, the deviation from the particle path is large for the initial position $(3.1,0)$. The particle path characteristics for frequency $f=1.0$ and amplitude $A=0.1$ is shown in figure 8 . It can be observed that the superimposition of this frequency is successful in making large number of particles that were initially moving around a single vortex to move around both vortices. It is clear from figures 6 and 8 that the frequency $f=1.0$ has significant influence on the periodic paths whose initial positions are near the elliptical point $(1.782,0)$. The variation of $T A D$ for frequency $f=1.0$ and for different amplitudes is shown in figure 9. It is seen that with increase in amplitude the effect of pulsations gets magnified. Hence, it can be said that the frequency of oscillation is the primary parameter influencing the deviation of particles from periodic paths and amplitude acts as a magnification factor.

The characteristics of particle motion for frequency $f=$ 1.0 and amplitude $A=0.5$ is shown in figure 10. In figure 10a, it can be seen that all the particle paths corresponding to all the initial conditions along $x$-axis revolve around both vortices. It is also clear from figure 9 that $T A D$ acts as a good quantitative measure to access the influence of superimposed pulsation. 

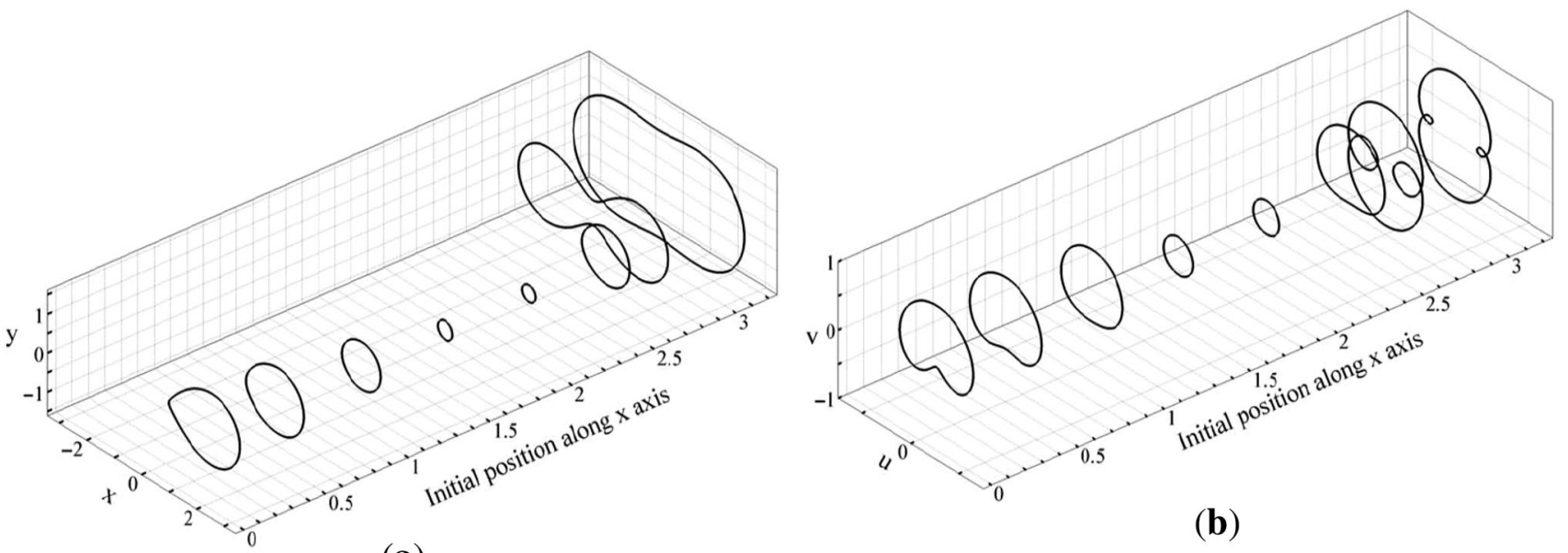

(a)

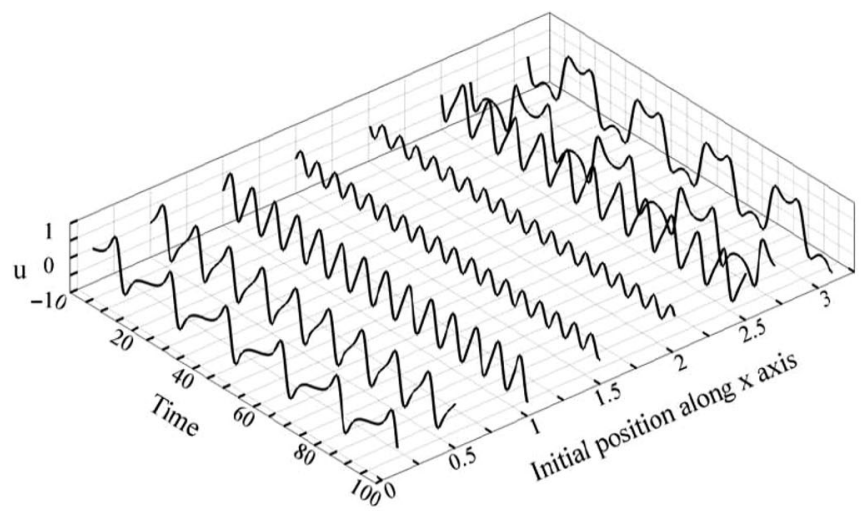

(c)

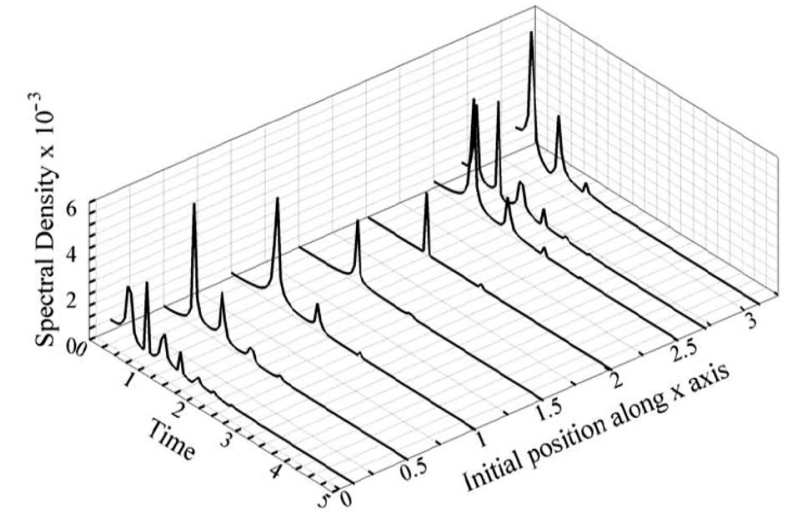

(d)

Figure 5. (a) Particle paths, (b) Lissajous curves, (c) signals of $u$-velocity, and (d) Fourier spectra of the $u$-velocity signal; for the pulsating case of co-rotating Lamb-Oseen vortices.

Table 2. Lowest dominant frequency for co-rotating vortices.

\begin{tabular}{llc}
\hline$x$ & $y$ & Lowest dominant frequency \\
\hline 0.1 & 0 & 0.37 \\
0.5 & 0 & 0.62 \\
1.0 & 0 & 0.94 \\
1.5 & 0 & 1.19 \\
2.01 & 0 & 1.19 \\
2.5 & 0 & 0.81 \\
2.7 & 0 & 0.31 \\
3.1 & 0 & 0.31 \\
\hline
\end{tabular}

\subsection{Counter-rotating vortices}

5.2a Counter-rotating vortices without pulsation: In the case of counter-rotating vortices in absence of pulsation, there exist two EPs as shown in table 3. From linear analysis the nature of the EPs are ascertained and both are found to be centers.

The particle paths shown in the figure 11 are similar to the streamlines in a counter-rotating vortex pair as documented in the texts of hydrodynamics [1]. Since flow due to stationary vortices is steady the particle paths are similar to streamlines. In the phase portrait of figure 11, yaxis is referred to as 'parting line' because a fluid particle originally on this line will neither deviate to right nor to left. The particles do not cross the parting line in case of non-pulsatile stationary vortices. However, in presence of pulsation, there is possibility of particles crossing the parting line that has been illustrated in later part of present work. All the other particle paths follow nearly circular trajectories with the ones closer to the parting line having larger radius. The particles near the elliptic points $( \pm 2.195$, $0)$ traverse a path with smaller radius.

Figure 12 shows the behavior of particle motion for nonpulsatile counter-rotating vortices. For the initial positions $(0.1,0)$ and $(0.5,0)$, the particle paths do not close during the time considered for computation $(T=100)$. These are the points that are close to the parting line and therefore are actually traversing a circular trajectory with larger radius.

5.2b Counter-rotating vortices with pulsation: As explained before, $T A D$ shows a quantitative measure of the effect 


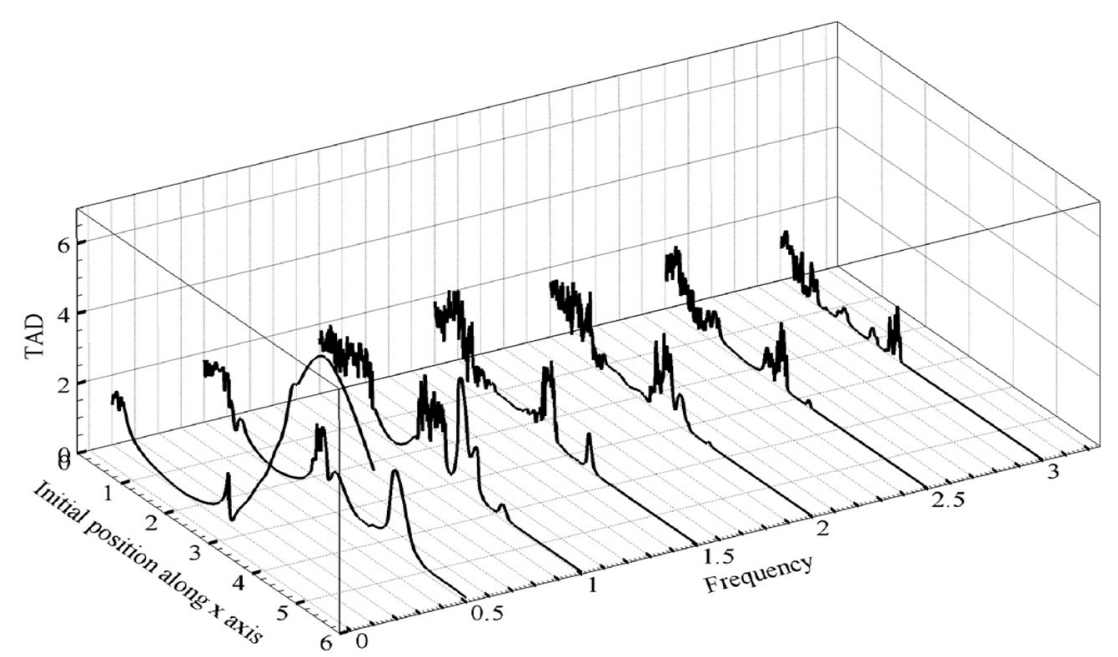

Figure 6. Variation of $T A D$ with initial position on $x$-axis for different frequencies and fixed amplitude $A=0.1$, for co-rotating LambOseen vortices.

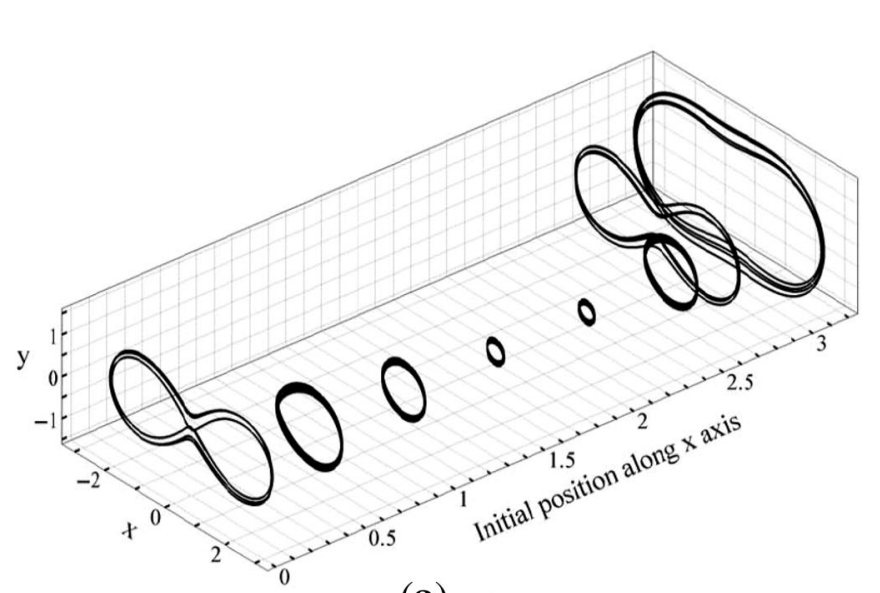

(a)

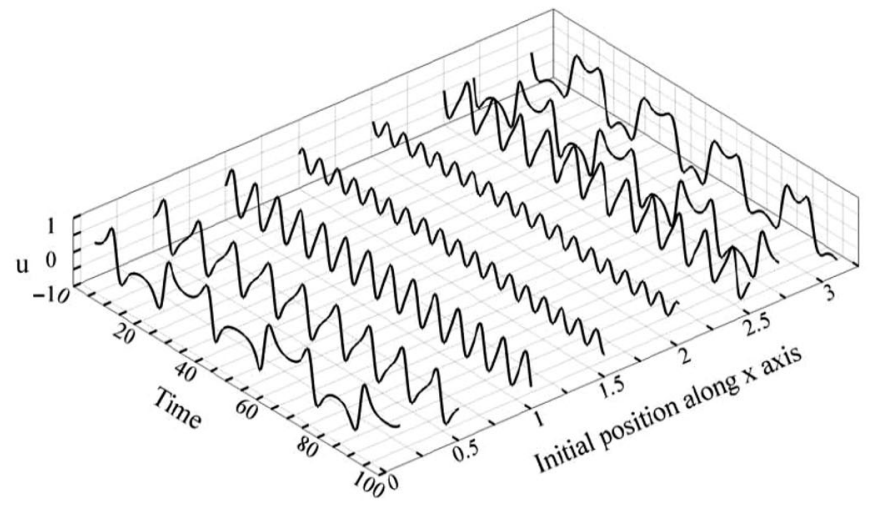

(c)

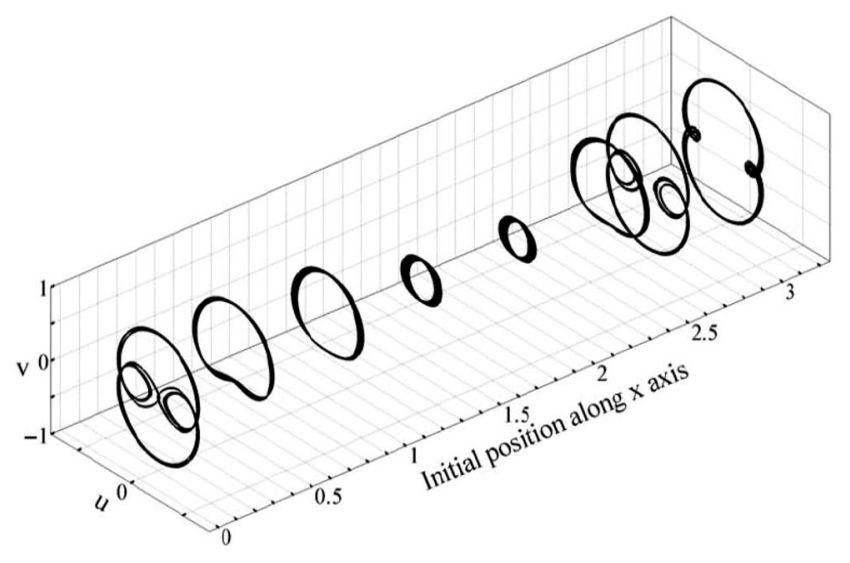

(b)

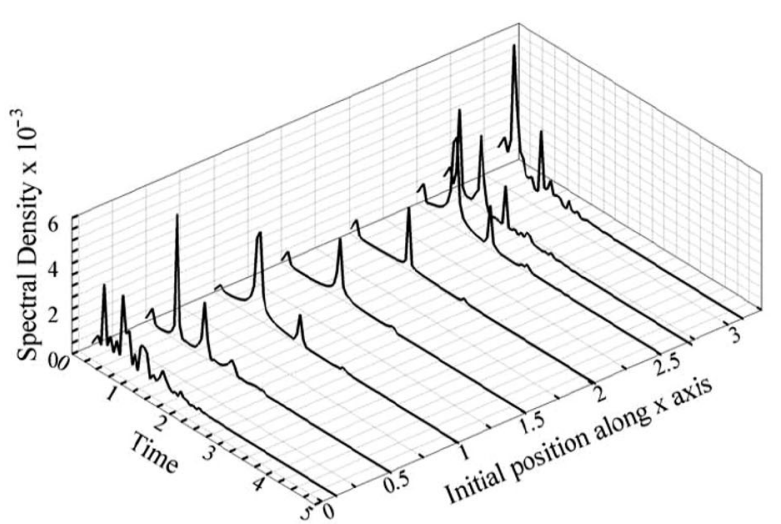

(d)

Figure 7. (a) Particle paths, (b) Lissajous curves, (c) signals of $u$-velocity, and (d) Fourier spectra of the $u$-velocity signal; for the pulsating case of co-rotating Lamb-Oseen vortices with $A=0.1, f=0.1$. 


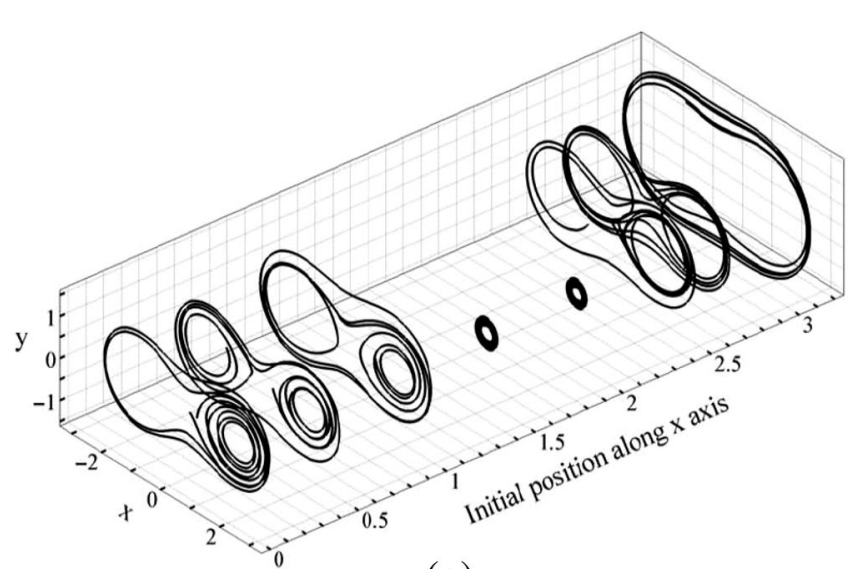

(a)

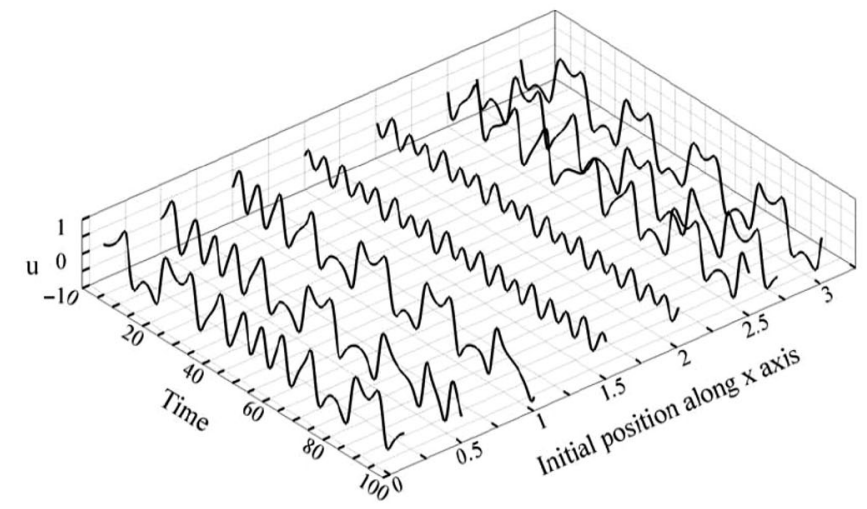

(c)

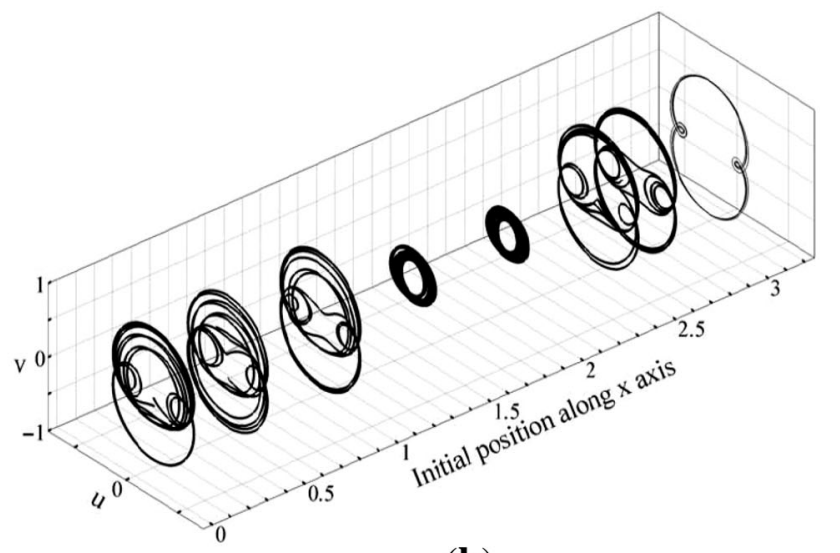

(b)

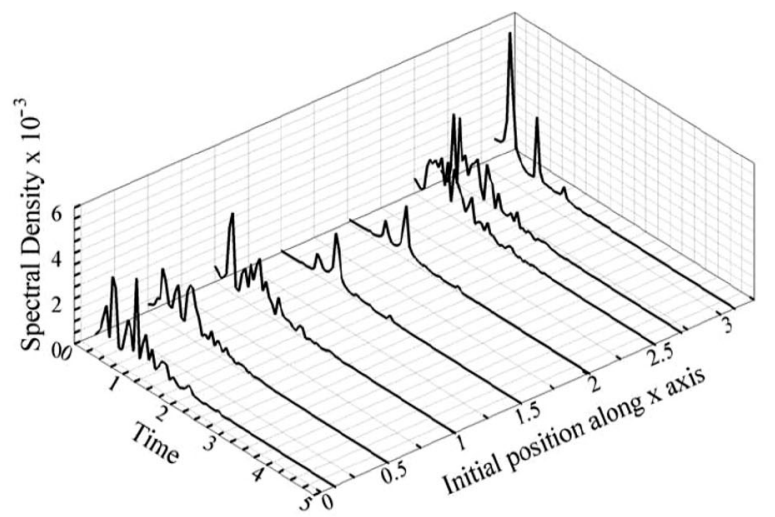

(d)

Figure 8. (a) Particle paths, (b) Lissajous curves, (c) signals of $u$-velocity, and (d) Fourier spectra of the $u$-velocity signal; for the pulsating case of co-rotating Lamb-Oseen vortices with $A=0.1, f=1.0$.

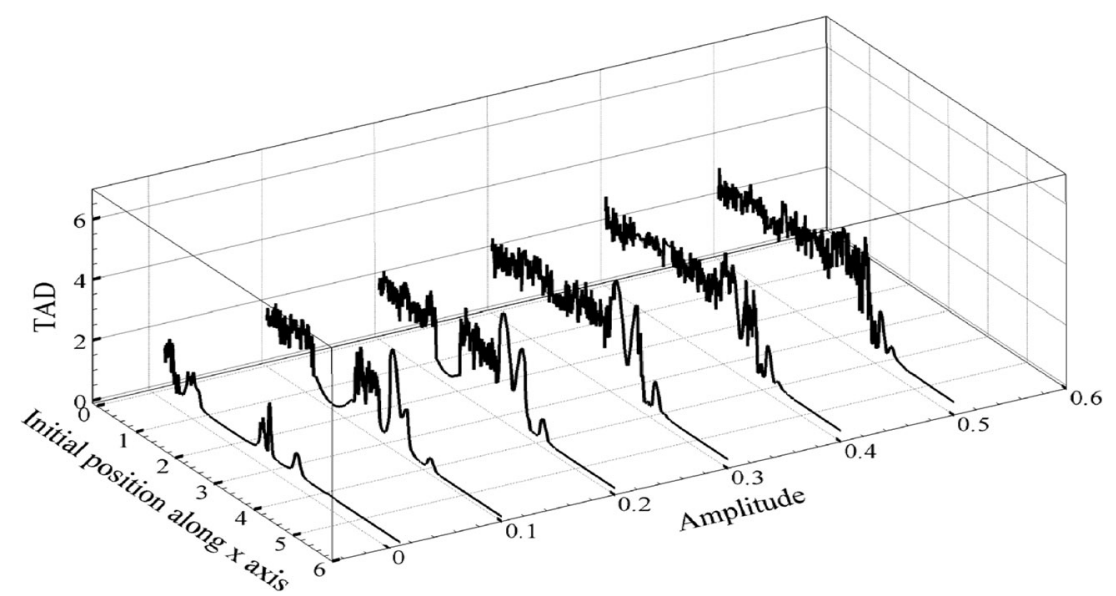

Figure 9. Variation of $T A D$ with particle initial positions on the $x$-axis for different amplitudes and fixed frequency of $f=1.0$, for the case of co-rotating Lamb-Oseen vortices. 

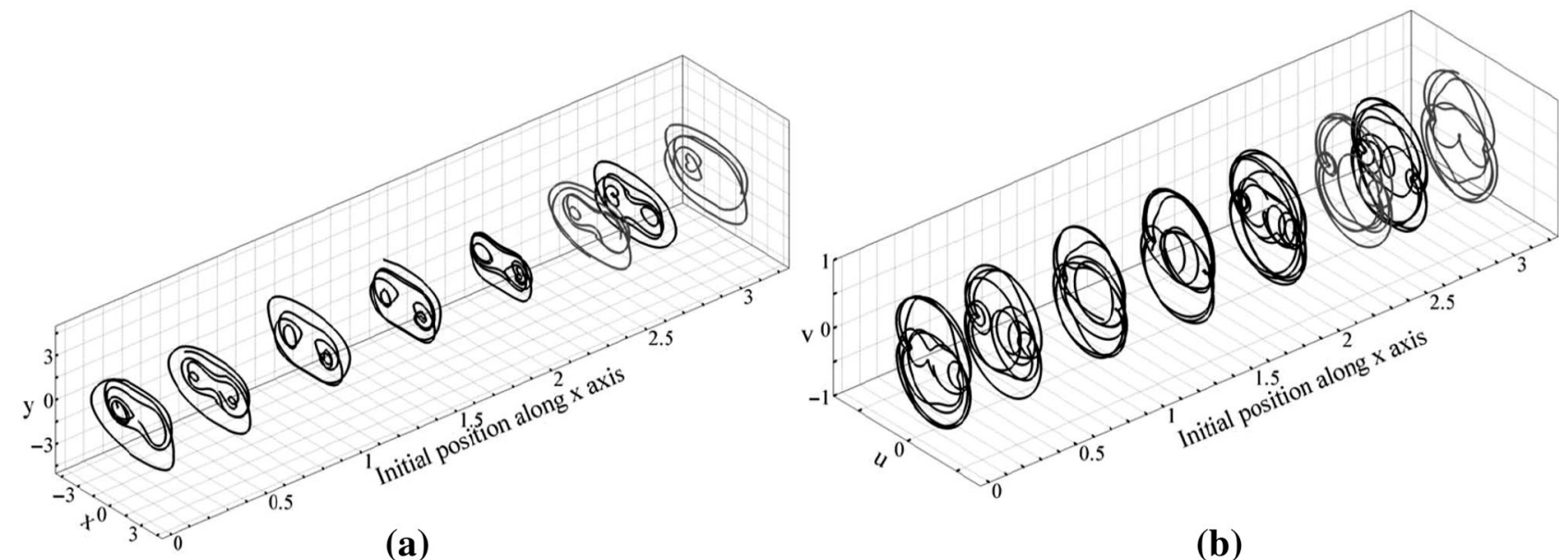

(b)

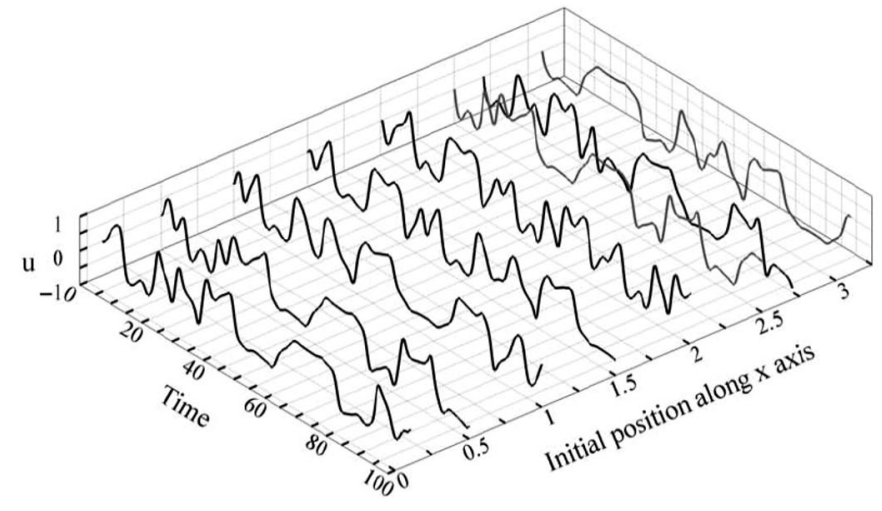

(c)

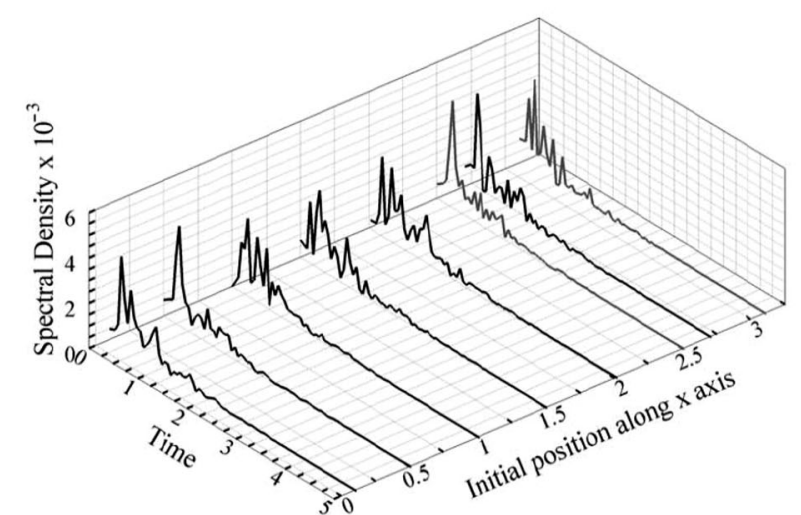

(d)

Figure 10. (a) Particle paths, (b) Lissajous curves, (c) signals of $u$-velocity, and (d) Fourier spectra of the $u$-velocity signal; for the pulsating case of co-rotating Lamb-Oseen vortices with $A=0.5, f=1.0$.

Table 3. Location of EPs and their nature for counter-rotating vortices in absence of pulsation.

Counter-rotating case: $\alpha=1$ and $\beta=-1$

\begin{tabular}{lcc}
\hline EPs,$\left(x_{e q}, y_{e q}\right)$ & Eigen values & Nature of the EP \\
\hline$(-2.195,0)$ & $\pm 1.19 i$ & Center \\
$(2.195,0)$ & $\pm 1.19 i$ & Center \\
\hline
\end{tabular}

produced by external pulsation on particle motion in a stationary vortex system. Figure 13 shows the variation of $T A D$ for different frequencies when the amplitude is $A=$ 0.1 for initial positions of particles on the positive $x$-axis Also, it is seen from figure 13 that for initial position of particles near the elliptic point $(2.195,0), T A D$ has lower values for low frequencies $(f=0.1$ and $f=0.5)$ and high frequencies $(f=2.5$ and $f=3.0)$. The values of TAD are higher near the elliptic point for frequency $f=1.0$. This can be attributed to some kind of in-phase superposition of velocity signals with applied pulsating signals. For all frequencies, TAD is larger for particle positions near the saddle. Similarly higher values of TAD are observed for particles with initial positions located at far right of the elliptic point. This is because the particle that starts near the saddle point moves in a path that takes it to the location farright of the elliptic point. The reason for this large deviation from periodic paths is evident from figure 14 which shows the particle motion characteristics for frequency $f=0.1$ and amplitude $A=0.1$. The large values of $T A D$ for particles near the parting line seem to have been caused due to increase in curvature of the particle trajectory. Also, it can be seen from figure 14a that the particle paths do not show much deviation for paths near the elliptic point.

As is clear from figure 13 that frequency $f=1.0$ produces large values of $T A D$ near the elliptic point. The 


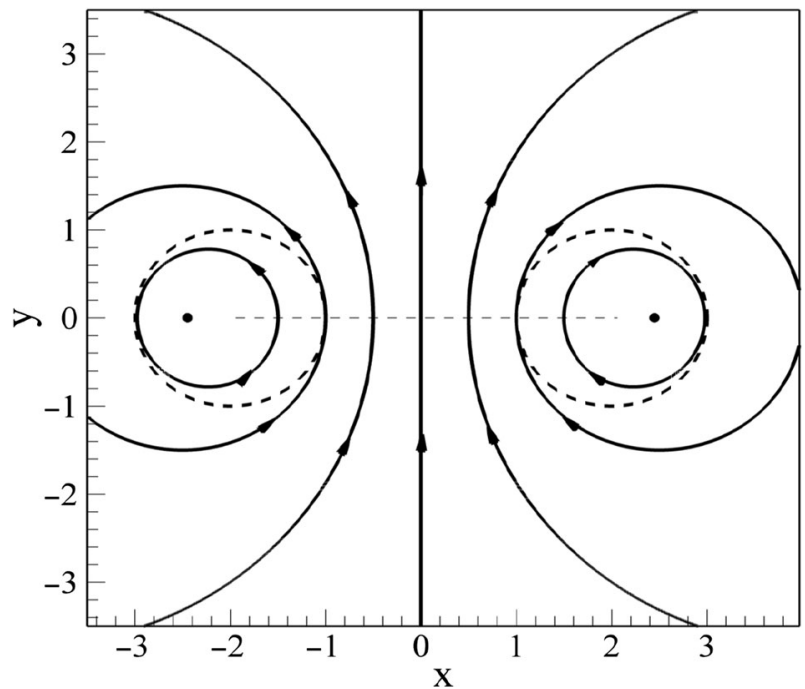

Figure 11. Phase portrait showing the particle paths for counterrotating Lamb-Oseen vortices. variation of $T A D$ with amplitude for fixed frequency of 1.0 is shown in figure 15. As in the co-rotating case it is seen that the values of $T A D$ increase with increase in amplitude. Hence, it is inferred that the amplitude plays the role of enhancing the value of $T A D$. The particle motion characteristics for superimposed pulsation of amplitude $A=0.5$ and frequency $f=1.0$ has been shown in figure 16. Larger deviations appear in particle paths for all the values of initial position as compared to the non-pulsatile case as well as compared to the case with amplitude $A=0.1$ and $f=0.1$.

In figure 13 and figure 15 sharp fluctuations in TAD can be seen. Figure 17 shows the variation of the particle paths and corresponding $u-v$ plots (Lissajous curves) for initial particle positions of $(4.97,0)$ and $(5.07,0)$ that are on the trough and crest of one such fluctuation in TAD for the superimposed pulsation of $A=0.1$ and $f=1.0$ given in figure 15. It is observed that for small deviation in initial positions, the particle paths deviate by a significant amount.
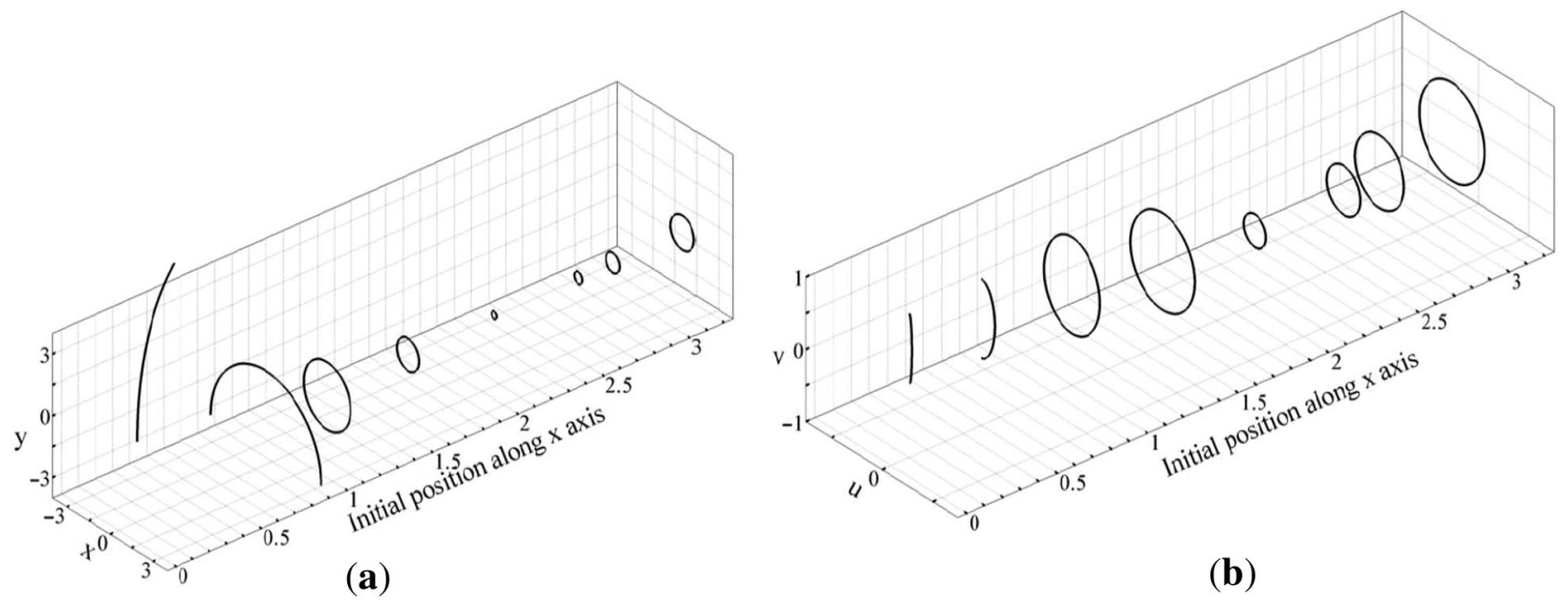

(b)

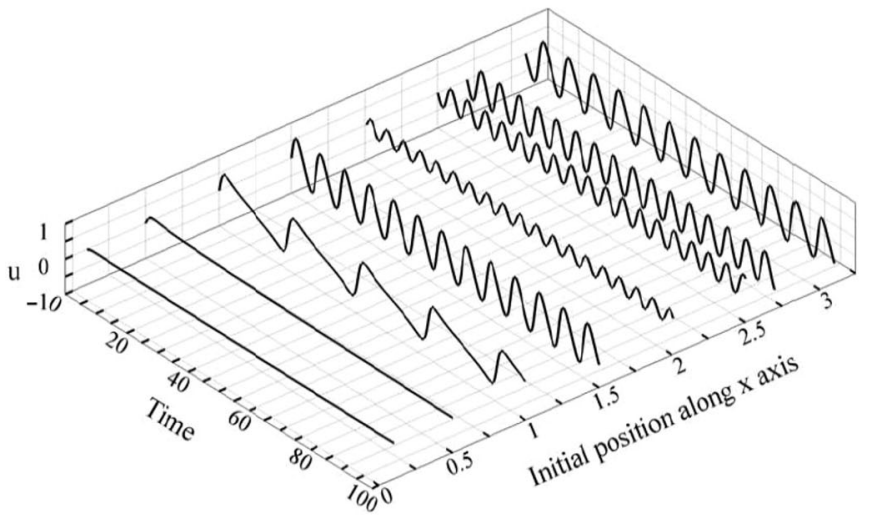

(c)

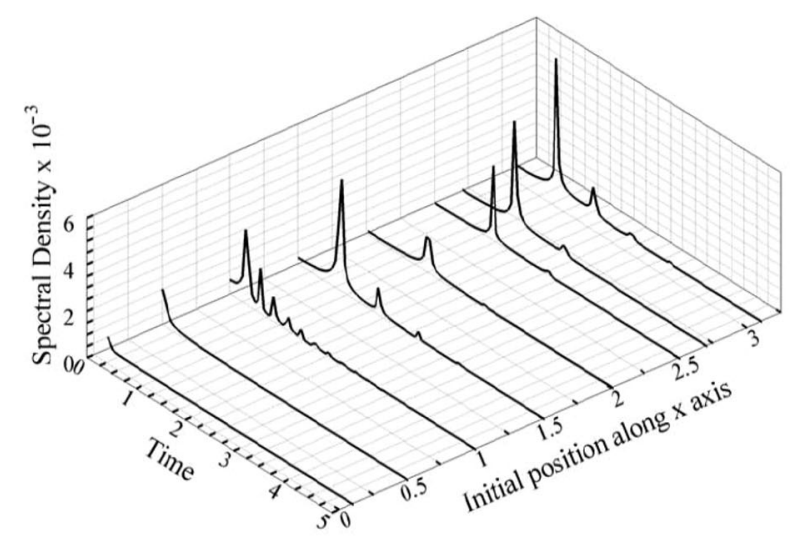

(d)

Figure 12. (a) Particle paths (b) Lissajous curves (c) signals of $u$-velocity and (d) Fourier spectra of the $u$-velocity signal; for the nonpulsating case of counter-rotating Lamb-Oseen vortices. 


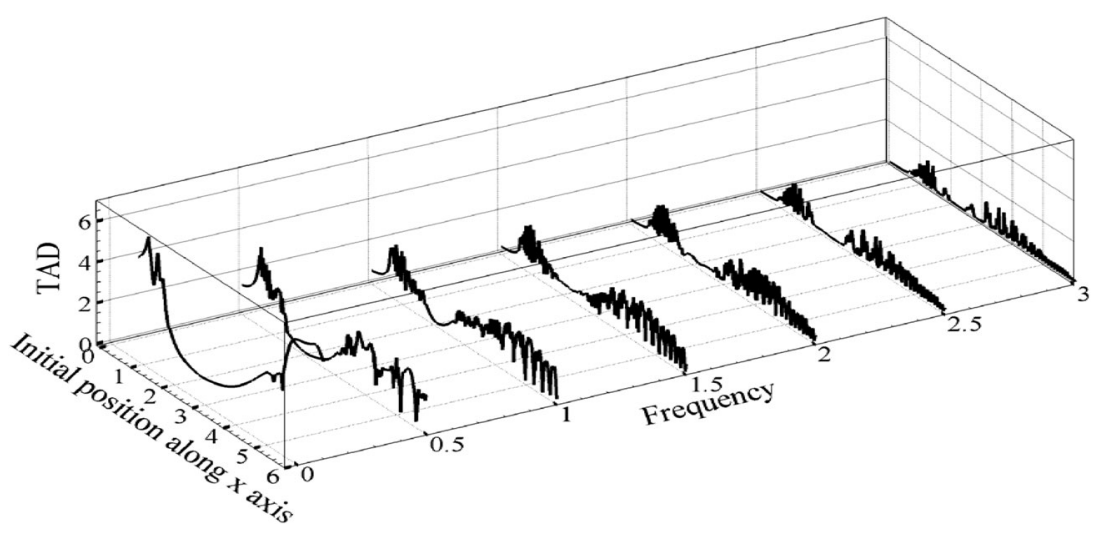

Figure 13. Variation of $T A D$ with initial position of the particle being on $x$-axis for different frequencies and fixed amplitude $A=0.1$, for the case of counter-rotating Lamb-Oseen vortices.

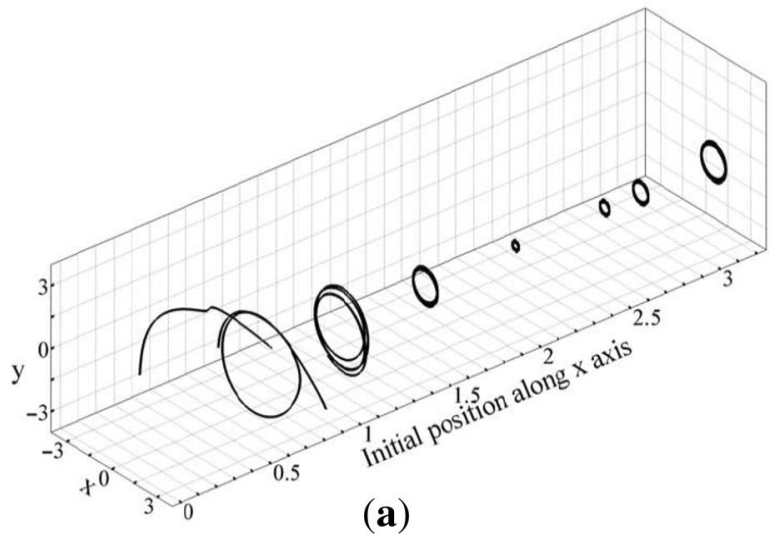

(a)

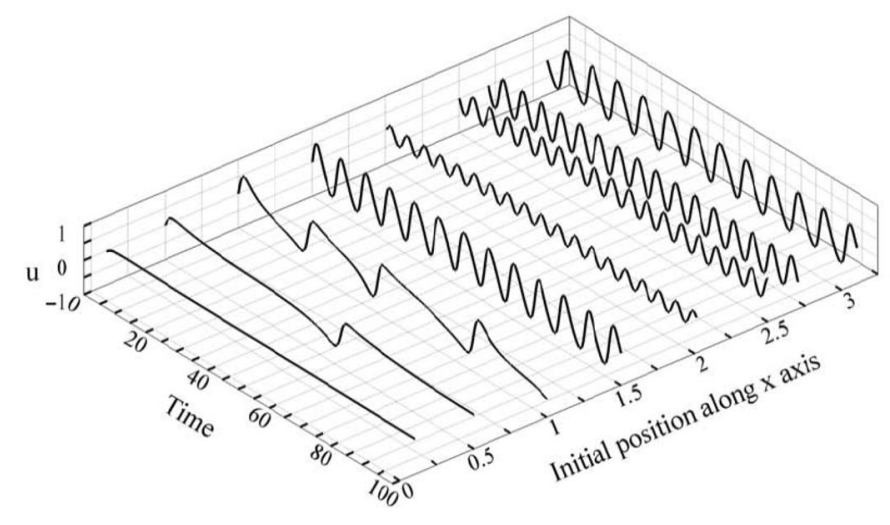

(c)

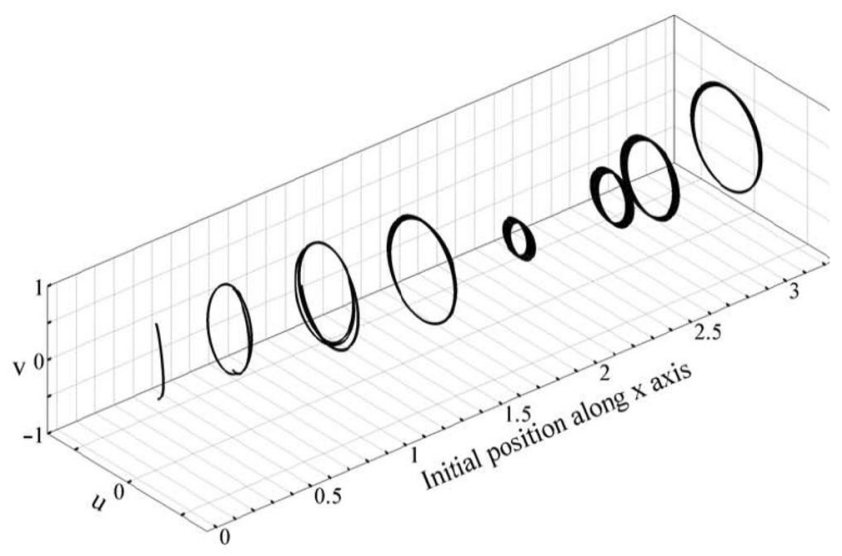

(b)

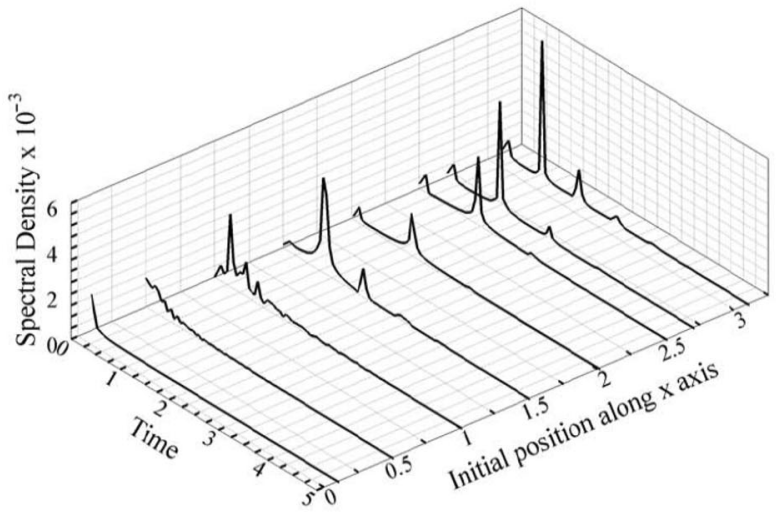

(d)

Figure 14. (a) Particle paths, (b) Lissajous curves, (c) signals of $u$-velocity, and (d) Fourier spectra of the $u$-velocity signal; for the pulsating case of co-rotating Lamb-Oseen vortices with $A=0.1, f=0.1$.

This can be considered as a case of sensitivity to initial conditions (SIC) that is beneficial when mixing is desired in the flow domain.

It was observed from figure 13 that the $T A D$ is highest near the parting lines at low frequency $f=0.1$. The variation of $T A D$ with increasing amplitude for frequency $f=0.1$ is shown in figure 18. It is seen that the variation becomes larger with higher values of amplitude. It is observed that the value of $T A D$ is almost doubled for all amplitude ranges when compared with the case of 


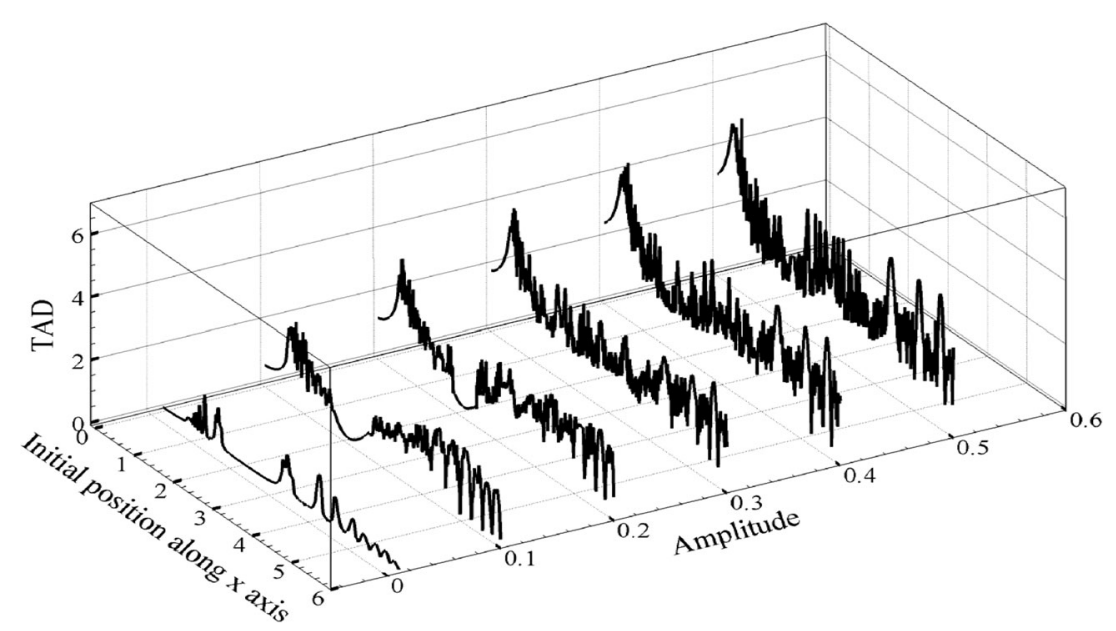

Figure 15. Variation of $T A D$ with initial position of particle on $x$-axis for different amplitudes and fixed frequency of $f=1.0$ in the case of counter-rotating Lamb-Oseen vortices.
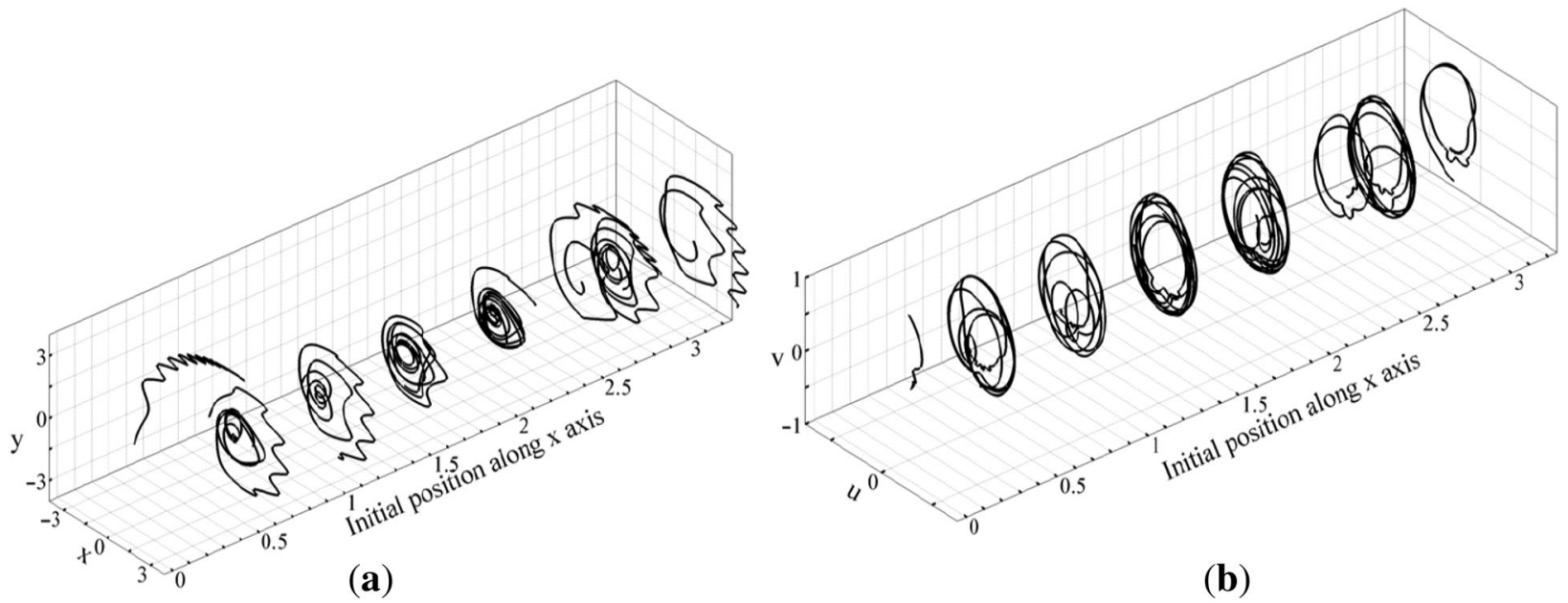

(b)

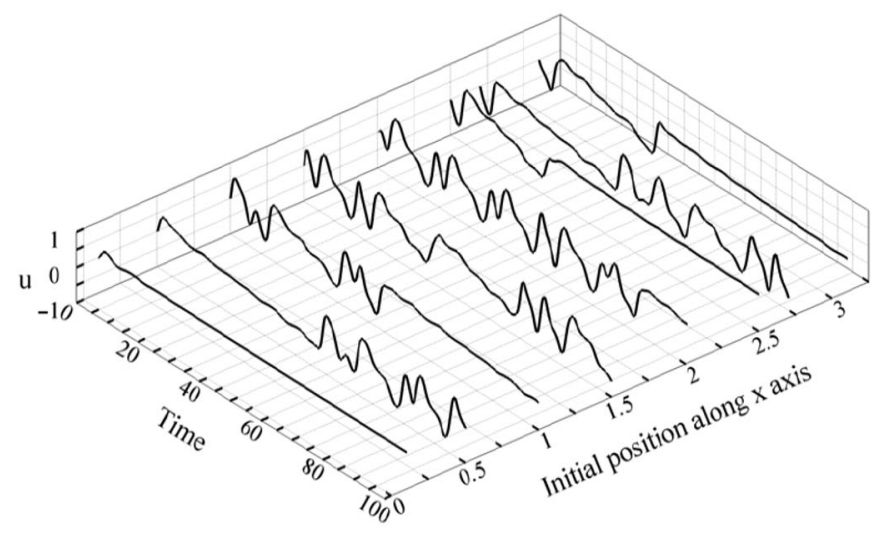

(c)

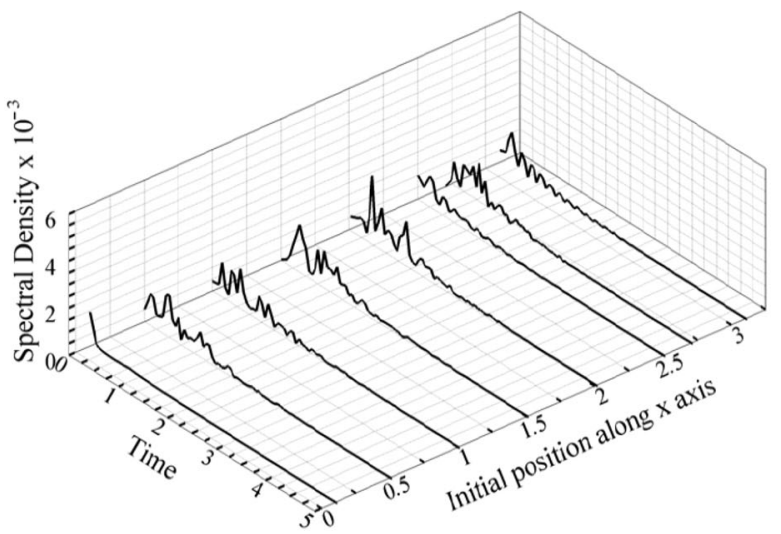

(d)

Figure 16. (a) Particle paths (b) Lissajous curves (c) signals of $u$-velocity and (d) Fourier spectra of the $u$-velocity signal; for the pulsating case of co-rotating Lamb-Oseen vortices with $A=0.5, f=1.0$. 

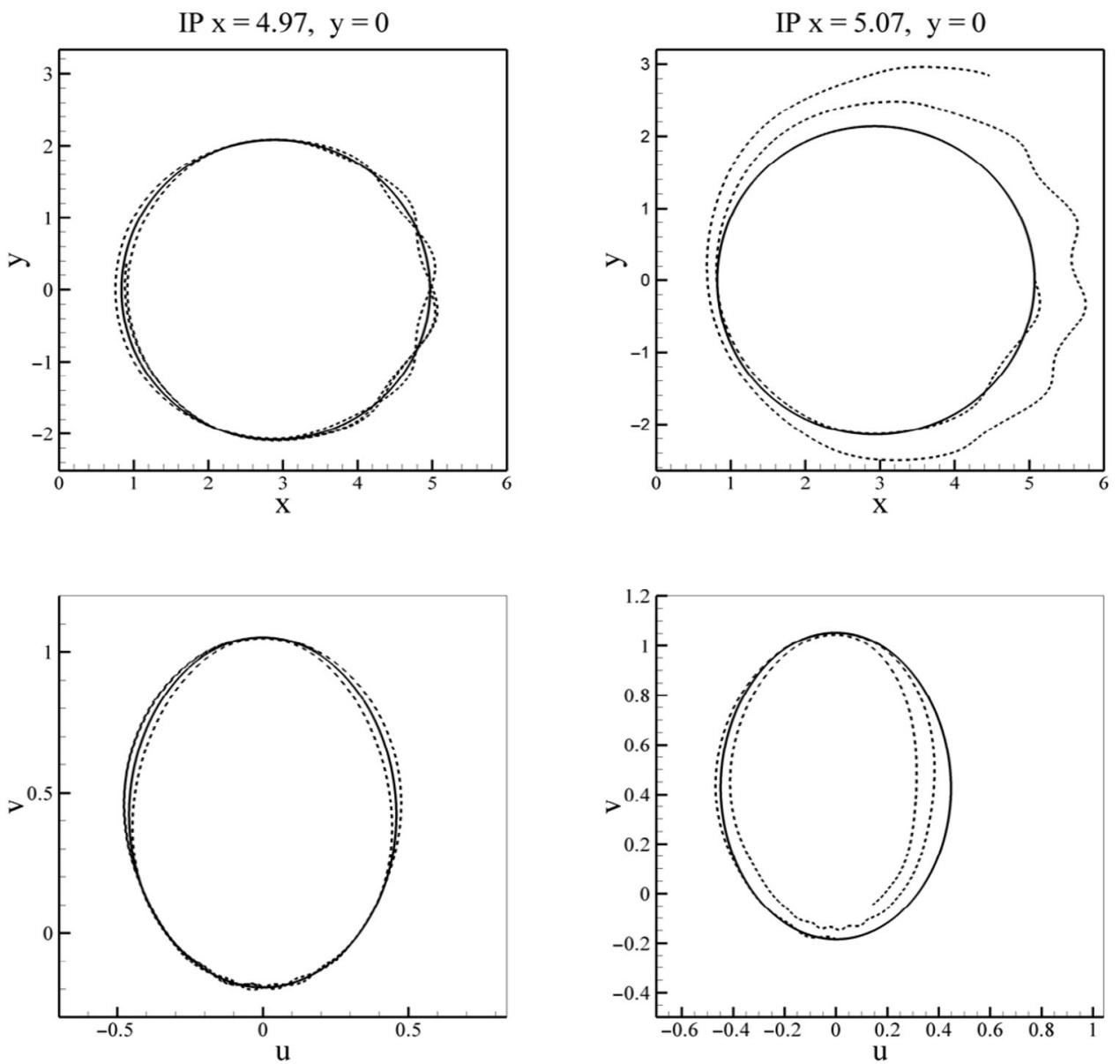

Figure 17. Variation of (a) paths and (b) $u-v$ plots (Lissajous curves) due to pulsation of $A=0.1$ and $f=1.0$ for initial positions (4.97, $0)$ and $(5.07,0)$ (curves with pulsation are shown by dashed line and without pulsations by continuous lines).

frequency $f=1.0$ shown in the figure 15 . To explore the possible reason for this, particle motion characteristics are plotted for frequency $f=0.1$ and amplitude $A=0.5$ in the figure 19. It is observed that the particle paths cross the parting line to get influenced by the other vortex and this is the reason for large $T A D$ as compared to the case of $A=$ 0.5 and $f=1.0$, where the particles revolve around only one vortex.

\section{Conclusions}

The effect of pulsation on a pair of stationary Lamb-Oseen vortices has been studied. The motion of particles is modeled as a dynamical system. If the core radius shows a small change, then the particle paths are almost periodic and can demonstrate higher degree of wandering in the presence of external pulsation. The deviation in particle path due to superposition of pulsation has been quantified using the concept of 'total average deviation' (TAD). For both corotating and counter-rotating stationary vortices, it is found that frequency of pulsation plays an important role in moving the particles out of periodic paths and amplitude enhances the wandering of particles. The saddle point that exists between the co-rotating vortices plays an important role in enhancing the influence of pulsations. Since the velocities near the saddle point are small, even a pulsation of low amplitude is capable enough to make the particle revolve around both the vortices. The fluid particles away from the vortex cores, but not in between them, show high $T A D$ for low frequencies. In the case of counter-rotating vortices, superposition of pulsation of high amplitude and low frequency helps the particle to cross the parting line which increases $T A D$ by a significant amount. 


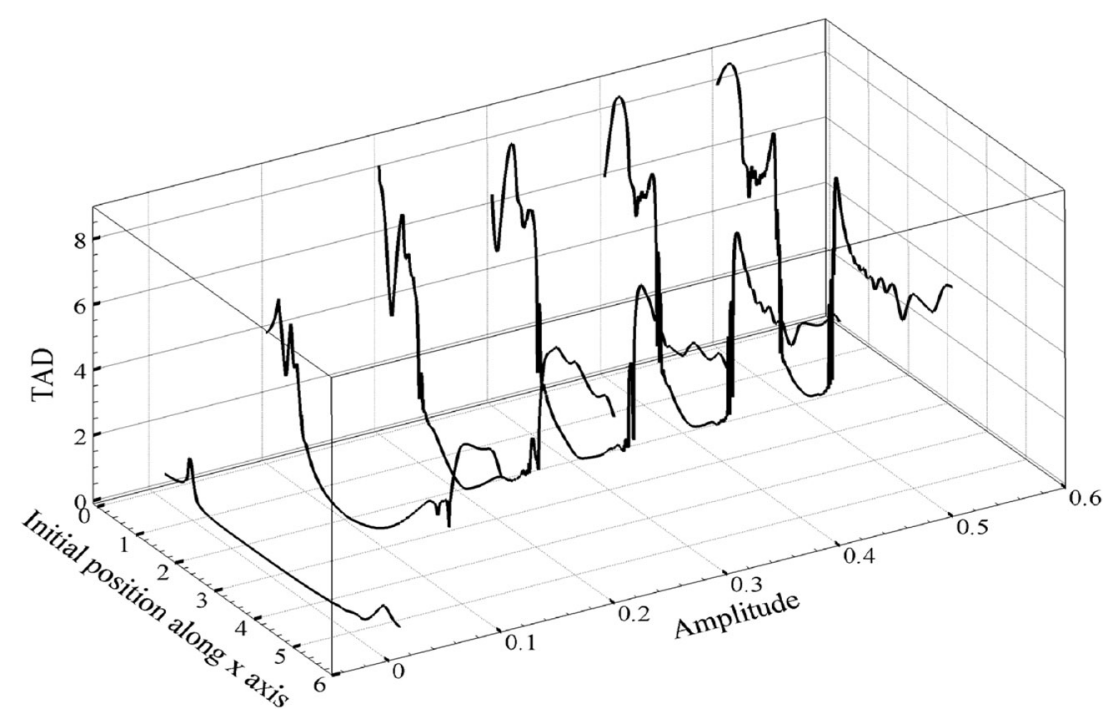

Figure 18. Variation of $T A D$ with particle initial position on the $x$-axis for different amplitudes for frequency for $f=0.1$ in the case of counter-rotating Lamb-Oseen vortices.
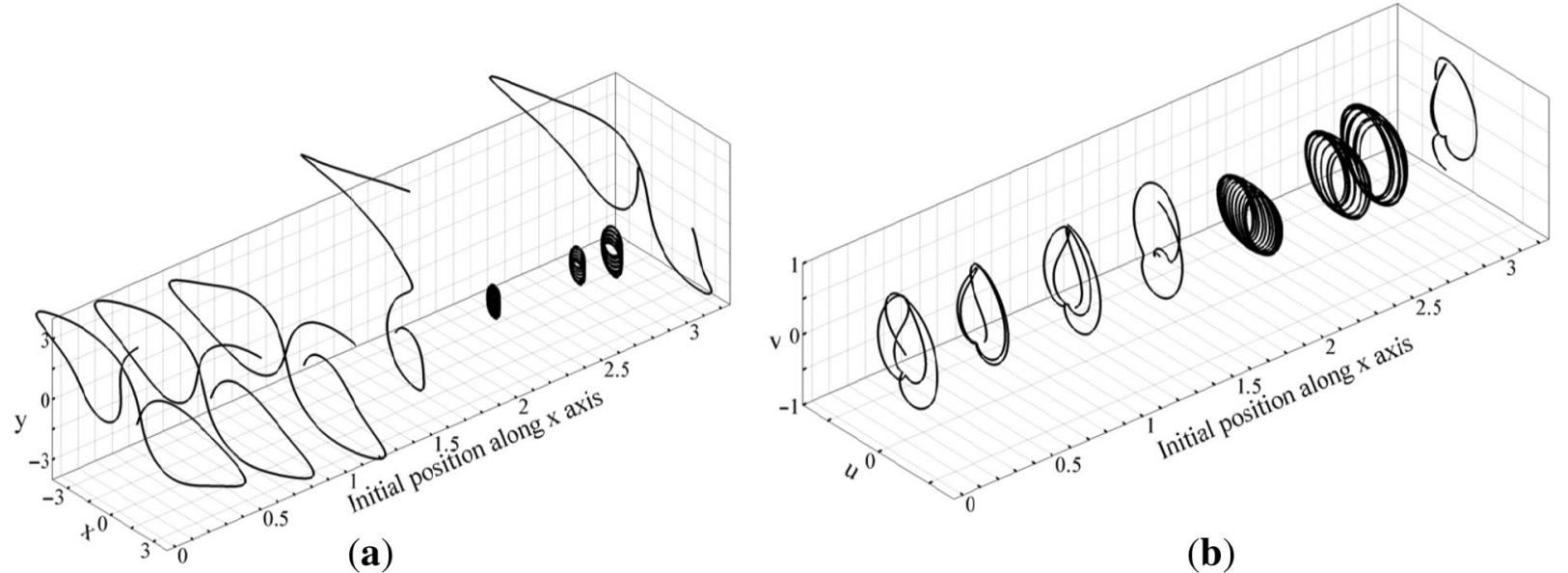

(b)

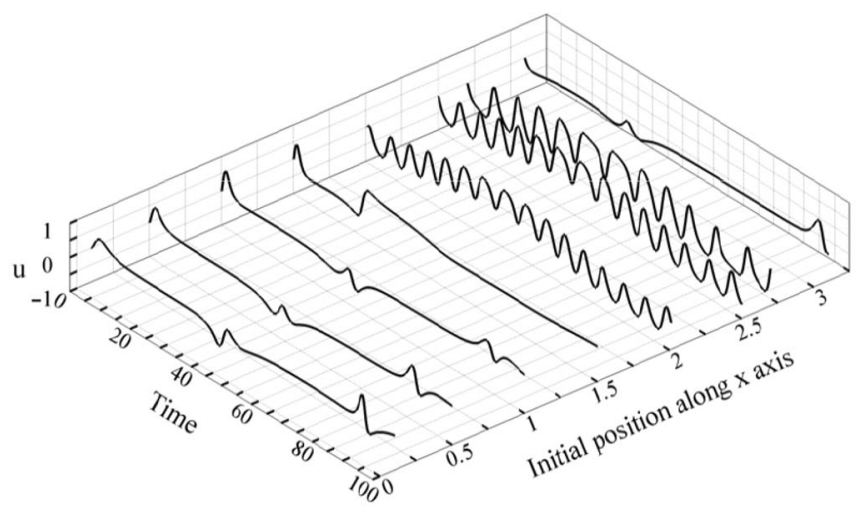

(c)

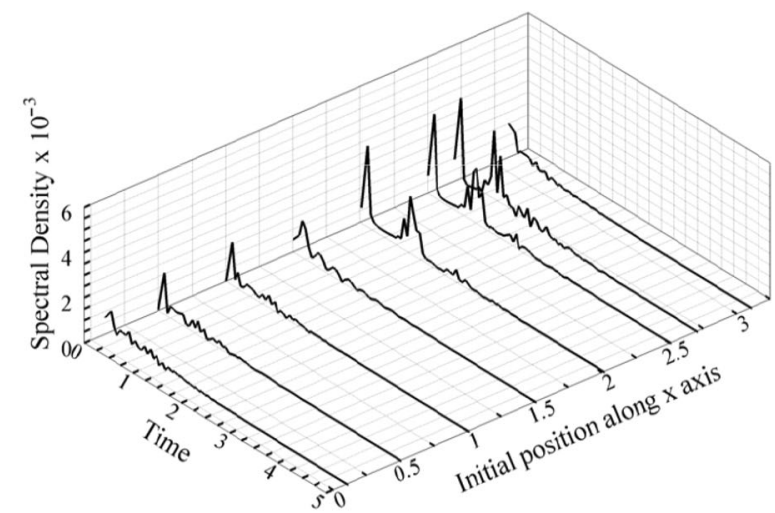

(d)

Figure 19. (a) Particle paths, (b) Lissajous curves, (c) signals of $u$-velocity, and (d) Fourier spectra of the $u$-velocity signal; for the pulsating case of co-rotating Lamb-Oseen vortices with $A=0.5, f=0.1$. 


\section{References}

[1] Lamb H 1932 Hydrodynamics. Cambridge University Press

[2] Aref H, Rott N and Thomann H 1992 Grobli's solution of the three vortex problem. Annu. Rev. Fluid Mech. 24: 1-20

[3] Batchelor G K 1967 An introduction to fluid dynamics. Cambridge: Cambridge University Press

[4] P G Saffman 1992 Vortex dynamics. Cambridge: Cambridge University Press

[5] Romero-Méndez R, Sen M, Yang K T and McClain R L 1998 Enhancement of heat transfer in an inviscid-flow thermal boundary layer due to a Rankine vortex. Int. J. Heat Mass Transf. 41: 3829-3840. doi:10.1016/S00179310(98)00094-5

[6] Jayavel S, Patil P P and Tiwari S 2008 Interaction of a skewed Rankine vortex pair. Phys. Fluids 20: 083601. doi:10.1063/1.2969115

[7] Jayavel S and Tiwari S 2012 Advection transport model of vortex enhanced heat transfer. Int. J. Heat Mass Transf. 55: 3273-3287. doi:10.1016/j.ijheatmasstransfer.2012.02.033

[8] Schaefer J W and Eskinazi S 1959 An analysis of the vortex street generated in a viscous fluid. J. Fluid Mech. 6: 241-260. doi: $10.1017 /$ S0022112059000593

[9] Jiménez J, Moffatt H K and Vasco C 1996 The structure of the vortices in freely decaying two-dimensional turbulence. J. Fluid Mech. 313: 209-222. doi:10.1017/ S0022112096002182

[10] Melander M V, Zabusky N J and Mcwilliams J C 1988 Symmetric vortex merger in two dimensions: causes and conditions. J. Fluid Mech. 195: 303-340. doi:10.1017/ S0022112088002435

[11] Brandt L K and Nomura K K 2006 The physics of vortex merger: further insight. Phys. Fluids 18: 051701. doi:10. $1063 / 1.2201474$
[12] Brandt L K, Cichocki T K and Nomura K K 2010 Asymmetric vortex merger: mechanism and criterion. Theor. Comput. Fluid Dyn. 24:163-167. doi:10.1007/s00162-0090154-1

[13] Aref H 1979 Motion of three vortices. Phys. Fluids 22: 393-400. doi:10.1063/1.862605

[14] Aref H, Stremler M A and Ponta F L 2006 Exotic vortex wakes-point vortex solutions J. Fluids Struct. 22: 929-940. doi:10.1016/j.jfluidstructs.2006.04.015

[15] Aref H and Pomphrey N 1982 Integrable and chaotic motions of four vortices. I. The case of identical vortices. Proc. $R$ Soc. Lond. A 380: 359-387

[16] Provenzale A 1999 Transport by coherent barotropic vortices. Annu. Rev. Fluid Mech. 31: 55-93. doi:10.1146/ annurev.fluid.31.1.55

[17] Ottino J M 1989 The kinematics of mixing: stretching, chaos, and transport. Cambridge: Cambridge University Press

[18] Hessel V, Löwe H and Schönfeld F 2005 Micromixers - a review on passive and active mixing principles. Chem. Eng. Sci. 60: 2479-2501. doi:10.1016/j.ces.2004.11.033

[19] Lu L and Papadakis G 2011 Investigation of the effect of external periodic flow pulsation on a cylinder wake using linear stability analysis. Phys. Fluids 23: 094105. doi:10. $1063 / 1.3625413$

[20] Newton P K 2001 The N-vortex problem analytical techniques. Berlin: Springer

[21] Meunier P, Ehrenstein U, Leweke T and Rossi M 2002 A merging criterion for two-dimensional co-rotating vortices. Phys. Fluids 14: 2757-2766. doi:10.1063/1.1489683

[22] Strogatz S H 1994 Nonlinear dynamics and chaos. Cambridge: Westview Press

[23] Hirsch M W, Smale S and Devaney R L 2004 Differential equations, dynamical systems and introduction to chaos. Amsterdam: Elsevier Academic Press 